\title{
Nondegenerate two-photon absorption in GaAs/AIGaAs multiple quantum well waveguides
}

\author{
Nicholas Cox $\odot,{ }^{1}$ Junxiong Wei $\odot,{ }^{2}$ Himansu Pattanaik, ${ }^{1, *}$ Thamer Tabbakh,,${ }^{1,3}$ Simon-Pierre Gorza, ${ }^{2}$ \\ David Hagan $1,{ }^{1, \dagger}$ and Eric W. Van Stryland ${ }^{1}$ \\ ${ }^{1}$ CREOL, The College of Optics and Photonics, University of Central Florida, Orlando, Florida 32816, USA \\ ${ }^{2}$ OPERA-Photonique, Université libre de Bruxelles (ULB), 50 Avenue F. D. Roosevelt, CP 194/5, 1050 Brussels, Belgium \\ ${ }^{3}$ King Abdulaziz City for Science and Technology, Riyadh 12354, Saudi Arabia
}

(Received 7 December 2019; revised manuscript received 18 January 2020; accepted 22 January 2020; published 30 March 2020)

\begin{abstract}
We present femtosecond pump-probe measurements of the nondegenerate (1960-nm excitation and 11761326-nm probe) two-photon absorption spectra of 8-nm GaAs/12 nm $\mathrm{Al}_{0.32} \mathrm{Ga}_{0.68}$ As quantum well waveguides. Experiments were performed with light pulses copolarized normal and tangential to the quantum well plane. The results are compared to perturbative calculations of transition rates between states determined by the $\mathbf{k} \cdot \mathbf{p}$ method with an 8- or 14-band basis. We find excellent agreement between theory and experiment for normal polarization, then use the model to support predictions of orders-of-magnitude enhancement of nondegenerate two-photon absorption as one constituent photon energy nears an intersubband resonance.
\end{abstract}

DOI: 10.1103/PhysRevResearch.2.013376

\section{INTRODUCTION}

Nondegenerate two-photon absorption (ND-2PA) is a process whereby absorption of an optical field is induced by a second, high irradiance field at a different wavelength. Applications of ND-2PA include detection [1], imaging [2], and all-optical switching [3]. Inverting carrier populations can also transform ND-2PA into nondegenerate two-photon gain [4-6], which is critical for realizing a two-photon semiconductor laser [7-9]. Waveguides are especially interesting for nonlinear optical applications because they enable strong effects through long interaction lengths. Group velocity mismatch (GVM) induced walkoff usually limits nondegenerate interactions, but dispersion engineering can mitigate or remove this walkoff entirely [10].

The degenerate 2PA (D-2PA) spectrum of infinite quantum wells was predicted by Spector [11] and Pasquarello and Quattropani [12]. Shortly after, Nithisoontorn et al. [13] experimentally demonstrated D-2PA to excitons in GaAs quantum wells. Shimizu [14] developed an excitonic model for D-2PA, and Tai et al. [15] verified their predictions with twophoton luminescence spectra. Later, Yang et al. [16] showed the anisotropy of D-2PA in quantum well waveguides.

Pasquarello and Quattropani [17] relaxed some of Shimizu's approximations and extended the analysis to nondegenerate photon pairs, predicting large enhancements as

\footnotetext{
*Present address: FARO Technologies, Londonderry, NH 03053 , USA.

†hagan@creol.ucf.edu

Published by the American Physical Society under the terms of the Creative Commons Attribution 4.0 International license. Further distribution of this work must maintain attribution to the author(s) and the published article's title, journal citation, and DOI.
}

one photon energy neared an intersubband resonance. Pattanaik et al. [18] quantitatively examined these nondegenerate resonance enhancements, expanding upon a six-band theory developed by Khurgin [19].

Here, we present pump-probe measurements of ND2PA coefficients in GaAs quantum wells. We studied 8-nm GaAs/12-nm $\mathrm{Al}_{0.32} \mathrm{Ga}_{0.68}$ As quantum wells at room temperature using beams polarized normal (TM-TM) and tangential (TE-TE) to the quantum well plane, and compared the results with a theoretical model for ND-2PA in finite wells neglecting excitonic effects.

We find that our perturbative model matches experimental results very closely for TM-TM beams, whereas a relatively large error in TE-TE predictions indicates that a more thorough analysis is needed. The TM-TM model shows that predictions of intersubband resonance enhancements of ND2PA also apply to finite wells, suggesting the possibility of extremely sensitive gated detection of subband-gap pulses [1].

This paper is organized as follows. In Sec. II, we derive a method for calculating ND-2PA coefficients in an arbitrary quasi-2D semiconductor. We introduce our GaAs quantum well waveguide in Sec. III and describe the pump-probe experiments carried out to find its ND-2PA coefficients. In Sec. IV, we apply the model of Sec. II to a simple quantum well structure that approximates our sample. Measurement results are presented in Sec. $\mathrm{V}$ followed by a discussion in Sec. VI.

In Appendix A, we give the equations and parameters used in numerical simulations of quantum well states and optical modes. Appendix B contains nonlinear wave propagation analysis, as well as the techniques used to match the calculation results to experimental curves. The rest of the Appendixes contain derivations and background for important equations: Appendix $\mathrm{C}$ gives a detailed derivation of ND-2PA coefficients in semiconductor quantum wells. Appendix D contains background about the Kane band-structure model 
for zinc-blende semiconductors, which is used in the envelope theory described in Appendix E. Appendix F shows the derivation of an intersubband matrix element in the envelope function expansion. Finally, an analytical equation for nonlinear propagation is derived in Appendix G.

\section{THEORETICAL BACKGROUND}

The $n$th level wave function of the $j$ th band (e.g., conduction, heavy hole, light hole) in a semiconductor confined in the $z$ direction is [20]

$$
\psi_{j n}\left(\mathbf{r} ; \mathbf{k}_{t}\right)=e^{i \mathbf{k}_{t} \cdot \mathbf{r}_{t}} F_{j n}\left(\mathbf{r}_{t}, z ; \mathbf{k}_{t}\right) .
$$

$F_{j n}$ is an envelope with lattice periodicity only in $\mathbf{r}_{t}$, the component tangential to the quantum well plane.

Second-order perturbation theory gives the net two-photon transition rate per unit volume [21],

$$
\begin{aligned}
W= & \frac{2 \pi}{\hbar} \frac{1}{V} \sum_{c v} \sum_{\mathbf{k}_{t}} \\
& \times\left|\sum_{i} \frac{\left\langle c\left|\hat{H}_{2}^{\prime}\right| i\right\rangle\left\langle i\left|\hat{H}_{1}^{\prime}\right| v\right\rangle}{E_{i v}\left(\mathbf{k}_{t}\right)-\hbar \omega_{1}}+\frac{\left\langle c\left|\hat{H}_{1}^{\prime}\right| i\right\rangle\left\langle i\left|\hat{H}_{2}^{\prime}\right| v\right\rangle}{E_{i v}\left(\mathbf{k}_{t}\right)-\hbar \omega_{2}}\right|^{2} \\
& \times \delta\left[E_{c v}\left(\mathbf{k}_{t}\right)-\hbar \omega_{1}-\hbar \omega_{2}\right],
\end{aligned}
$$

where $|c\rangle$ and $|v\rangle$ are conduction and valence envelopes, respectively. $H_{l}^{\prime}$ is the interaction Hamiltonian for a vector potential of magnitude $A_{0 l}$ and polarization $\hat{\mathbf{e}}_{l}$, given by [22]

$$
\hat{H}_{l}^{\prime}=\frac{e A_{0 l}}{2 m_{0}} \hat{\mathbf{e}}_{l} \cdot\left(\hat{\mathbf{p}}+\hbar \mathbf{k}_{t}\right) .
$$

The $\hbar \mathbf{k}_{t}$ term arises from the chain rule for the momentum operator $\hat{\mathbf{p}}=-i \hbar \nabla$ applied to states in the form of Eq. (1). This term is usually ignored because it frequently cancels out, but we leave it in for completeness.

The transition rate is converted to an ND-2PA coefficient by [23]

$$
\alpha_{2}\left(\omega_{1} ; \omega_{2}\right)=\frac{\hbar \omega_{1}}{2 I_{1} I_{2}} W
$$

which describes the attenuation of wave 1 at frequency $\omega_{1}$ induced by wave 2 at $\omega_{2} . I_{1}$ and $I_{2}$ are the incident field irradiances,

$$
I_{l}=\frac{1}{2} n_{l} c \epsilon_{0} \omega_{l}^{2}\left|A_{0 l}\right|^{2}
$$

with $n_{l}$ the effective index at $\omega_{l}$. We also introduce a unitless matrix element between envelopes [24],

$$
M_{j n, i m}^{(l)}\left(\mathbf{k}_{t}\right)=\frac{\hbar}{m_{0} P} \hat{\mathbf{e}}_{l} \cdot\left\langle F_{j n}\left|\mathbf{p}+\hbar \mathbf{k}_{t}\right| F_{i m}\right\rangle .
$$

As detailed in Appendix $\mathrm{D}$, the Kane parameter $P=$ $\hbar / m_{0}\left\langle i S\left|p_{x}\right| X\right\rangle$ is the optical coupling strength between conduction and valence bands.

Finally, we combine Eqs. (2)-(6) into a general expression for ND-2PA coefficients:

$$
\alpha_{2}\left(\omega_{1} ; \omega_{2}\right)=K \frac{E_{p}}{n_{1} n_{2} L_{z} E_{g}^{4}} f_{2}\left(\frac{\hbar \omega_{1}}{E_{g}} ; \frac{\hbar \omega_{2}}{E_{g}}\right),
$$

where $f_{2}$ is the dimensionless spectral function,

$$
\begin{aligned}
f_{2}\left(x_{1} ; x_{2}\right)= & \sum_{c v} \sum_{\kappa_{0}} \frac{1}{2 \pi} \int_{0}^{2 \pi} \kappa_{0}\left|\frac{\partial \epsilon_{c v}}{\partial \kappa}\right|_{\kappa_{0}}^{-1} \\
& \times\left|\sum_{i} \frac{M_{c i}^{(2)} M_{i v}^{(1)}}{\epsilon_{i v}-x_{1}}+\frac{M_{c i}^{(1)} M_{i v}^{(2)}}{\epsilon_{i v}-x_{2}}\right| d \phi .
\end{aligned}
$$

See Appendix C for a full derivation. The quantity $E_{g}$ is the band gap of the quantum well material and $E_{p}=2 m_{0} P^{2} / \hbar^{2}$ is the Kane energy. The parameter $L_{z}$ is the total thickness of the structure in the $z$ direction. For a single quantum well, $L_{z}$ is the sum of the barrier and well widths.

Equation (7) is valid in any unit system so long as the material-independent parameter $K$ is adjusted accordingly. With energies and lengths written in Hartree atomic units $\left[\hbar=m_{0}=e=1 /\left(4 \pi \epsilon_{0}\right)=1\right]$, this constant is simply $K=(\pi / c)^{2}=(\pi / 137)^{2}$. The final 2PA coefficient can then be converted to $\mathrm{cm} / \mathrm{GW}$ by the conversion factor $1 \mathrm{a} . \mathrm{u} .=$ $29.36 \mathrm{~cm} / \mathrm{GW}$.

The integral in Eq. (8) is taken over the azimuthal angle of $\mathbf{k}_{t}$, whose magnitude has been replaced by the unitless quantity $\kappa=k_{t} P / E_{g}$. Energies are also normalized by letting $\epsilon_{j k}=E_{j k} / E_{g}$ and $x_{j}=\hbar \omega_{j} / E_{g}$. Each $\kappa_{0}$ is a real, positive solution to

$$
\epsilon_{c v}(\kappa, \phi)-x_{1}-x_{2}=0 .
$$

We derived Eqs. (7) and (8) without making any assumptions of specific band-structure or layer design, leaving us with a general expression for ND-2PA coefficients in quasitwo-dimensional (2D) materials. Later, we make approximations to simplify calculations for the symmetric quantum wells introduced in the next section.

\section{SAMPLE AND EXPERIMENT}

We experimentally investigated GaAs quantum wells MBE-grown by Sandia National Laboratories on an $n$ GaAs (100) substrate. A waveguiding structure was formed in the $z$ direction by growing $2-\mu \mathrm{m}$-thick $\mathrm{Al}_{0.7} \mathrm{Ga}_{0.3} \mathrm{As}$ cladding layers on either side of a $2-\mu \mathrm{m}$ active region. The active region comprised 100 repetitions of $(8 \mathrm{~nm}$ $\mathrm{GaAs}) /\left(12 \mathrm{~nm} \mathrm{Al}_{0.32} \mathrm{Ga}_{0.68} \mathrm{As}\right)$ quantum wells, with barrier widths chosen so that coupling between wells is negligible. Transverse optical confinement was achieved by etching a 3- $\mu \mathrm{m}$-wide ridge through the active region and lower cladding. Finally, the sample was cleaved to a length of $3.6 \mathrm{~mm}$. The layer structure and geometry are seen in Fig. 1.

Figure 2 shows the optical setup employed to study the sample. A short-wavelength probe and long-wavelength pump came from the signal and idler, respectively, of a Spectra-Physics OPAL optical parametric oscillator (OPO) synchronously driven by a Spectra-Physics Tsunami Ti: $\mathrm{Al}_{2} \mathrm{O}_{3}$ laser with $82 \mathrm{MHz}$ repetition rate. We tuned the driving laser wavelength between 730 and $795 \mathrm{~nm}$ to study 2PA at sum photon energies near the absorption edge. For each driving laser wavelength, the OPO phase matching was adjusted to fix the idler at $1960 \mathrm{~nm}$. In effect, the pump was fixed at $1960 \mathrm{~nm}$ while the probe varied between 1176 and $1326 \mathrm{~nm}$. This pump photon energy was chosen to be below the D-2PA edge so that 


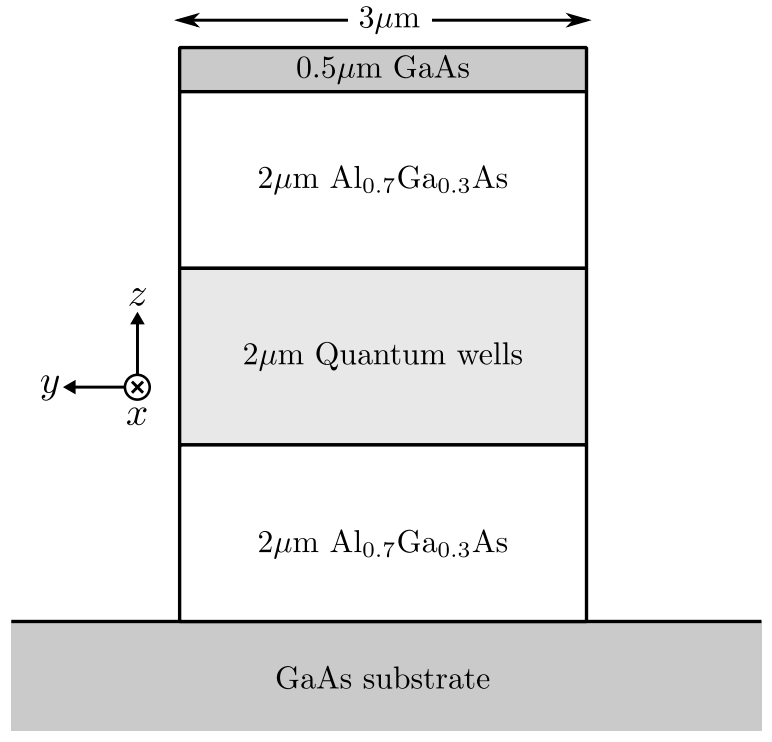

FIG. 1. A schematic of the quantum well waveguide samples studied. The $2-\mu \mathrm{m}$ quantum well region consists of $100 \times(8 \mathrm{~nm}$ $\mathrm{GaAs}) /\left(12 \mathrm{~nm} \mathrm{Al} \mathrm{l}_{0.32} \mathrm{Ga}_{0.68}\right)$ quantum wells.

two-photon photogenerated carriers did not interfere with data interpretation.

After fixing the signal and idler wavelengths, their polarizations were set to TE ( $y$ polarized) or TM ( $z$ polarized) using broadband half-wave plates. The probe then traveled through a delay line and combined with the pump at a dichroic mirror. The beams were end-fire coupled into the ridge waveguide (Fig. 1) using a microscope objective and collected by a lensed fiber at the exit facet. The lensed fiber was connected to a Yokogawa AQ6370D spectrum analyzer (OSA in Fig. 2) to compare the probe output power spectrum with and without the pump's presence. The process was repeated at a series of

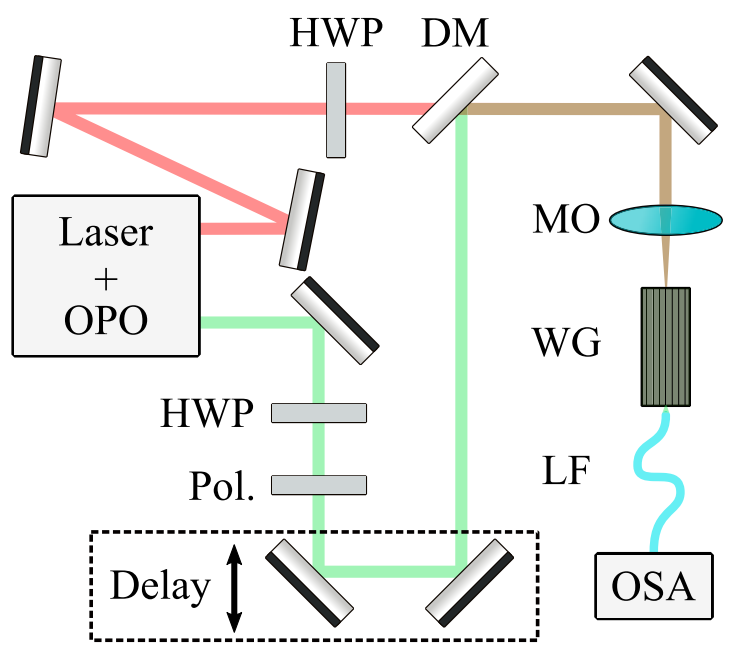

FIG. 2. A schematic of the experimental setup. OPO: optical parametric oscillator; HWP: half-wave plate; Pol: polarizer; DM: dichroic mirror; MO: microscope objective; WG: AlGaAs/GaAs quantum well waveguide; LF: lensed fiber; OSA: optical spectrum analyzer. The pump laser for the OPO is a Ti:sapphire laser which is tuned between 730 and $795 \mathrm{~nm}$. probe delays to generate curves of normalized transmission versus delay.

It is necessary to know the pump power at the facet inside the waveguide to convert normalized transmission to an ND2PA coefficient. This input power was calculated by backpropagating the OSA-measured output power to the front facet using

$$
P_{\text {out }}=P_{\text {in }} \exp (-\sigma L) T
$$

which depends on the waveguide loss $\sigma$, the sample propagation length $L$, and the the output facet coupling efficiency $T$. This efficiency was experimentally determined by temporarily replacing the microscope objective of Fig. 2 with a lensed fiber identical to the one at the output. By measuring $P_{\text {out }} / P_{\text {in }}$ for this symmetric system, we found the facet transmission by

$$
T=\left[\frac{P_{\text {out }}}{P_{\text {in }}} \exp (\sigma L)\right]^{1 / 2} \text {. }
$$

After finding a waveguide loss of $\sigma_{\mathrm{TM}}=0.46 \mathrm{~mm}^{-1}$ and $\sigma_{\mathrm{TE}}=0.56 \mathrm{~mm}^{-1}$ (see Appendix B 2), we determined the transmission coefficients of the front facet to be $T_{\mathrm{TM}}=0.47$ and $T_{\mathrm{TE}}=0.54$.

Autocorrelation measurements of the 1960-nm pump at four different sum wavelengths gave the following pulse widths: $227 \mathrm{fs}$ at $789 \mathrm{~nm}, 221 \mathrm{fs}$ at $774 \mathrm{~nm}, 156 \mathrm{fs}$ at $754 \mathrm{~nm}$, and $149 \mathrm{fs}$ at $745 \mathrm{~nm}$. By comparing to the measured spectra, we determined pump pulse widths to be an average of $9 \%$ greater than the Gaussian bandwidth limit.

\section{CALCULATION OF 2PA COEFFICIENTS}

This section describes the model used to calculate the 2PA coefficients of a symmetric GaAs quantum well. We begin by calculating the energy levels and envelope functions of each subband level. Then we construct expressions for the optical matrix elements between all states. Finally, Eq. (8) is used to calculate the 2PA coefficients for parabolic bands.

\section{A. Wave-function envelopes}

Calculation of ND-2PA coefficients requires knowledge of the energy levels and wave functions between which twophoton transitions occur. We begin by expanding the envelope functions in the basis of zone-center wave functions $u_{\nu 0}(\mathbf{r})$ $[25,26]$ :

$$
F_{j n}\left(\mathbf{r}, \mathbf{k}_{t}\right)=\sum_{\nu} \chi_{j n}^{v}\left(z ; \mathbf{k}_{t}\right) u_{\nu 0}(\mathbf{r}) .
$$

The basis is chosen to consist of either 8 or 14 spin-degenerate bands (see Appendix D for details). For ease of calculation, we only solve for envelopes at $\mathbf{k}_{t}=0$; the approximation applied for $\mathbf{k}_{t} \neq 0$ is discussed later in this subsection. Taking the alloy composition-dependent energy offset of band $j$ as a $z$-dependent potential $V_{j}(z)$ leads to a second-order Schrödinger equation [27],

$$
\frac{1}{2} p_{z} \frac{1}{m_{j}^{z}\left(E_{j n}, z\right)} p_{z} \chi_{j n}^{j}(z)+V_{j}(z) \chi_{j n}^{j}(z)=E_{j n} \chi_{j n}^{j}(z) .
$$

The superscript on $\chi_{j n}^{j}$ denotes the dominant envelope, and $m_{j}^{z}\left(E_{j n}, z\right)$ is the state's energy-dependent effective mass in the 
$z$ direction. Choosing the eight-band basis for Eq. (12) yields the effective-mass relations [27]

$$
\begin{aligned}
& \frac{m_{0}}{m_{c}^{z}(E, z)}= 1+\frac{m_{0}}{\mathscr{M}_{c c}^{z z}}+\frac{2}{3} \frac{E_{p}}{E-V_{l}(z)} \\
&+\frac{1}{3} \frac{E_{p}}{E+\Delta-V_{s}(z)}, \\
& \frac{m_{0}}{m_{l}^{z}(E, z)}=1+\frac{m_{0}}{\mathscr{M}_{l l}^{z z}}+\frac{2}{3} \frac{E_{p}}{E-V_{c}(z)-E_{g}}, \\
& \frac{m_{0}}{m_{h}^{z}(E, z)}=1+\frac{m_{0}}{\mathscr{M}_{h h}^{z z}} .
\end{aligned}
$$

See Appendix E for more information on the effective masses and the envelope Schrödinger equation. The quantity $\Delta$ is the spin-orbit split-off energy and $m_{0} / \mathscr{M}_{k k}^{z z}$ are remote band contributions included by Löwdin's perturbation method [28]. We take the approximation that this remote band contribution is independent of energy.

In the 14-band basis, the conduction-band effective mass changes to

$$
\begin{aligned}
\frac{m_{0}}{m_{c}^{z}(E, z)}= & 1+\frac{m_{0}}{\mathscr{M}_{c c}^{z z}}+\frac{2}{3} \frac{E_{p}}{E-V_{l}(z)}+\frac{1}{3} \frac{E_{p}}{E+\Delta-V_{s}(z)} \\
& +\frac{2}{3} \frac{E_{p}^{\prime}}{E-E_{g}^{\prime}-V_{l^{\prime}}(z)}+\frac{1}{3} \frac{E_{p}^{\prime}}{E-E_{s^{\prime}}-V_{s^{\prime}}(z)},
\end{aligned}
$$

where $l^{\prime}$ is the light electron band at $E=E_{g}^{\prime}$ and $s^{\prime}$ is the split-off electron band at $E=E_{s^{\prime}}$. $E_{p}^{\prime}$ is the coupling energy between the two sets of conduction bands. All hole effective masses are identical to the eight-band results.

If we set $V_{j}=0$ and $E=E_{j, \text { bulk }}$ in Eqs. (14) and (15), we find expressions for bulk GaAs effective masses. As was done in Ref. [29], we choose $m_{0} / \mathscr{M}_{k k}^{z z}$ so that calculated bulk effective masses match experimental values.

We assume the $\mathbf{k}_{t}$ band dispersion to have the form

$$
E_{j n}\left(\mathbf{k}_{t}\right)=E_{j n}(0)+\frac{\hbar^{2} k_{t}^{2}}{2 m_{j}^{t}},
$$

where $m_{j}^{t}$ is the effective mass in the transverse direction. This parabolic band approximation has been successfully used to calculate 2PA coefficients for bulk semiconductors [22], at the cost of ignoring some fine structure in the dispersion [24].

\section{B. Matrix elements}

Equipped with a model for electronic states, we can proceed to calculate the optical matrix elements between them. Two-photon transitions across the band gap always require an interband transition, for which we consider allowed and forbidden paths. For TM-TM polarizations, the remaining step is assumed to be an allowed intersubband transition. In TE-TE 2PA, the remaining step is assumed to be a forbidden self-transition [30]. These two interaction types vary differently with wavelength near the 2PA edge, causing anisotropy between the polarization schemes.

Intersubband matrix elements are calculated between the envelopes found from Eq. (13). As a consequence, the results are only strictly valid at the band edge. For TM polarization,
TABLE I. Optical matrix elements $M_{j n, i m}^{z}=\hat{\mathbf{z}} \cdot \mathbf{M}_{j n, i m}$. Only transitions to $\alpha$ spin states are shown since transitions to $\beta$ states can be found from the relation $M_{j n, i m}^{z}=\left(M_{i m, j n}^{z}\right)^{*}$.

\begin{tabular}{lccc}
\hline \hline & $c \alpha, n$ & $h \alpha, n$ & $l \alpha, n$ \\
\hline$c \alpha, m$ & Eq. (17) & 0 & $\sqrt{\frac{2}{3}} \cos \theta \delta_{n m}$ \\
$c \beta, m$ & 0 & $-\frac{1}{\sqrt{2}} \sin \theta \delta_{n m}$ & $\frac{1}{\sqrt{6}} \sin \theta \delta_{n m}$ \\
$h \alpha, m$ & 0 & Eq. (17) & 0 \\
$h \beta, m$ & $-\frac{1}{\sqrt{2}} \sin \theta \delta_{n m}$ & 0 & 0 \\
$l \alpha, m$ & $\sqrt{\frac{2}{3}} \cos \theta \delta_{n m}$ & 0 & Eq. (17) \\
$l \beta, m$ & $\frac{1}{\sqrt{6}} \sin \theta \delta_{n m}$ & 0 & 0 \\
\hline \hline
\end{tabular}

we find that

$$
M_{j n, i m}^{z}=\frac{\hbar}{2 P}\left\langle\chi_{j n}^{j}\left|\frac{1}{m_{i}^{\prime}} p_{z}+p_{z} \frac{1}{m_{j}^{\prime}}\right| \chi_{i m}^{j}\right\rangle \delta_{i j},
$$

where

$$
\frac{1}{m_{k}^{\prime}}=\frac{1}{m_{k}(E, z)}-\frac{1}{\mathscr{M}_{k k}^{z z}} .
$$

See Appendix F for justification of the above equation. This form exhibits two improvements over that in Refs. [18] and [19], viz. $M_{j n, i m}^{z}=\hbar /\left(m_{j} P\right)\left\langle\chi_{j n}^{j}\left|p_{z}\right| \chi_{j m}^{j}\right\rangle$. The first is to include energy scaling of the effective mass, and the second is to account for the fact that interband coupling depends only on the inverse effective-mass component arising from interactions within the basis. Equation (17) can also be compared to that in Ref. [31], which ignores the subtraction of remote band contributions.

Self-transitions are forbidden because they describe optical coupling between states of nearly identical symmetry. Equation (17) with $m=n$ shows that these contributions are negligible for TM polarization. For TE fields we use the relation $\langle\mathbf{p}\rangle=\left(m_{0} / \hbar\right) \nabla_{\mathbf{k}} E\left(\mathbf{k}_{t}\right)-\hbar \mathbf{k}_{t}$ [32] with the energy given by Eq. (16). Fixing TE polarization in the $y$ direction leads to

$$
M_{j n, i m}^{y}=\frac{\hbar^{2}}{m_{j}^{t} P} k_{t} \sin \phi \delta_{m n} \delta_{i j} .
$$

Equation (17) is also valid for interband transitions, but using it would ignore the $\mathbf{k}_{t}$ dependence once again. Instead, we use the method of Yamanishi and Suemune [33] to estimate matrix elements from the bulk band structure:

$$
M_{j n, i m}^{(l)}=\frac{\hbar}{m_{0} P} \hat{\mathbf{e}}_{l} \cdot\left\langle u_{j 0}(\mathbf{r})|\hat{\mathbf{p}}| u_{i 0}(\mathbf{r})\right\rangle^{\prime} \delta_{n m},
$$

where $u_{j 0}(\mathbf{r})$ is the zone-center basis function for band $j$. As described in Appendix D, the prime denotes that the basis functions are rotated by an angle $\theta=\cos ^{-1}\left[k_{z} /\left(k_{z}^{2}+k_{t}^{2}\right)^{1 / 2}\right]$. We find $k_{z}$ from the energy relation $E_{j n}(0)=\hbar^{2} k_{z}^{2} / 2 m_{j}^{z}$. Equation (20) applies to TM and TE polarizations and accounts for allowed $(\propto \cos \theta)$ and forbidden $(\propto \sin \theta)$ transitions.

All matrix elements for both polarizations are compiled in Tables I and II, in a similar form to those in Refs. [21,24]. 
TABLE II. Optical matrix elements $M_{j n, i m}^{y}=\hat{\mathbf{y}} \cdot \mathbf{M}_{j n, i m}$. Only transitions to $\alpha$ spin states are shown since transitions to $\beta$ states can be found from the relation $M_{j n, i m}^{y}=\left(M_{i m, j n}^{y}\right)^{*}$.

\begin{tabular}{lccc}
\hline \hline & $c \alpha, n$ & $h \alpha, n$ & $l \alpha, n$ \\
\hline$c \alpha, m$ & $\frac{m_{0}}{m_{c}^{t}} \frac{2 \kappa}{\epsilon_{p}} \sin \phi \delta_{n m}$ & 0 & $\sqrt{\frac{2}{3}} \sin \theta \sin \phi \delta_{n m}$ \\
$c \beta, m$ & 0 & 0 & $-\frac{1}{\sqrt{6}}(\cos \theta \sin \phi+i \cos \phi) \delta_{n m}$ \\
$h \alpha, m$ & 0 & $\frac{m_{0}}{m_{h}^{t}} \frac{2 \kappa}{\epsilon_{p}} \sin \phi \delta_{n m}$ & 0 \\
$h \beta, m$ & $\frac{1}{\sqrt{2}}(\cos \theta \sin \phi-i \cos \phi) \delta_{n m}$ & 0 & 0 \\
$l \alpha, m$ & $\sqrt{\frac{2}{3} \sin \theta \sin \phi \delta_{n m}}$ & 0 & $\frac{m_{0}}{m_{l}^{t}} \frac{2 \kappa}{\epsilon_{p}} \sin \phi \delta_{n m}$ \\
$l \beta, m$ & $-\frac{1}{\sqrt{6}}(\cos \theta \sin \phi+i \cos \phi) \delta_{n m}$ & 0 \\
\hline \hline
\end{tabular}

\section{2PA coefficients for parabolic bands}

The energy separation between parabolic conduction and valence bands is given in normalized form by

$$
\epsilon_{c v}(\kappa)=\epsilon_{c v}(0)+\frac{m_{0}}{\mu_{c v}^{t}} \frac{\kappa^{2}}{\epsilon_{p}},
$$

where $\epsilon_{p}=E_{p} / E_{g}$ and $1 / \mu_{c v}^{t}=1 / m_{c}^{t}-1 / m_{v}^{t}$. We see immediately from Eq. (21) that

$$
\left|\frac{\partial \epsilon_{c v}}{\partial \kappa}\right|_{\kappa_{0}}^{-1}=\frac{\mu_{c v}^{t}}{m_{0}} \frac{\epsilon_{p}}{2 \kappa_{0}} .
$$

Combining Eqs. (9) and (21), we find

$$
\kappa_{0}=\left\{\frac{\mu_{c v}^{t}}{m_{0}} \epsilon_{p}\left[x_{1}+x_{2}-\epsilon_{c v}(0)\right]\right\}^{1 / 2} .
$$

Feeding the results of Eqs. (21)-(23) into Eq. (8) gives a general expression for the dimensionless scaling factor in the parabolic band approximation:

$$
\begin{aligned}
f_{2}\left(x_{1} ; x_{2}\right)= & \epsilon_{p} \sum_{c \alpha, v} \frac{\mu_{c v}^{t}}{m_{0}} \Theta\left[x_{1}+x_{2}-\epsilon_{c v}(0)\right] \\
& \times \frac{1}{2 \pi} \int_{0}^{2 \pi}\left|\sum_{i} \frac{M_{c i}^{(2)} M_{i v}^{(1)}}{\epsilon_{i v}-x_{1}}+\frac{M_{c i}^{(1)} M_{i v}^{(2)}}{\epsilon_{i v}-x_{2}}\right|^{2} d \phi .
\end{aligned}
$$

The sum runs only over $\alpha$ conduction states, with identical $c \beta$ transitions included by a factor of 2 . The step function $\Theta$ defines the range where the solution of Eq. (23) is real. The aforementioned angular rotation factor in the interband matrix element is found to be

$$
\cos \theta=\left(1+\frac{\mu_{c v}^{t}}{m_{c}^{z}} \frac{x_{1}+x_{2}-\epsilon_{c v}(0)}{\epsilon_{c}(0)}\right)^{-1 / 2} .
$$

Two-photon absorption coefficients can finally be found from Eq. (7). Note that each quantum well is treated as a separate system so that $L_{z}$ in this equation is the total thickness of a single well $(20 \mathrm{~nm})$.

Equation (24) is valid for TM-TM, TE-TE, and the mixed-polarization TE-TM configurations. Because the copolarized schemes provide sufficient information about the ND-2PA anisotropy, we do not perform the less tractable cross-polarized calculations. The next two subsections provide some simplifications for the TM-TM and TE-TE cases.

\section{TM-TM 2PA coefficients}

As shown in Fig. 3, every TM-TM two-photon transition includes one interband and one intersubband transition. Because envelope parity alternates with subband index, the required TM matrix element [Eq. (17)] imposes the selection rule $n-m=2 k+1$ for integer $k$. Since every element in Table I is independent of $\phi$, the azimuthal integral of Eq. (24) reduces to unity such that

$$
\begin{aligned}
f_{2}\left(x_{1} ; x_{2}\right)= & \frac{\epsilon_{p}}{x_{1} x_{2}^{2}} \sum_{c \alpha, v} \frac{\mu_{c v}^{t}}{m_{0}}\left|\sum_{i} \frac{M_{c i}^{z} M_{i v}^{z}}{\epsilon_{i v}-x_{1}}+\frac{M_{c i}^{z} M_{i v}^{z}}{\epsilon_{i v}-x_{2}}\right|^{2} \\
& \times \Theta\left[x_{1}+x_{2}-\epsilon_{c v}(0)\right] .
\end{aligned}
$$

Both matrix elements are nonzero at the ND-2PA edge for doubly allowed transitions, giving the steplike shape characteristic of linear quantum well absorption.

\section{TE-TE 2PA coefficients}

Per Fig. 3, each TE-TE 2PA path involves an interband and a self transition. Two-photon transitions therefore inherit the selection rules of the the interband transition, namely $m=n$. Using the matrix elements from Table II, which are generally $\phi$ dependent, we simplify the dimensionless scaling

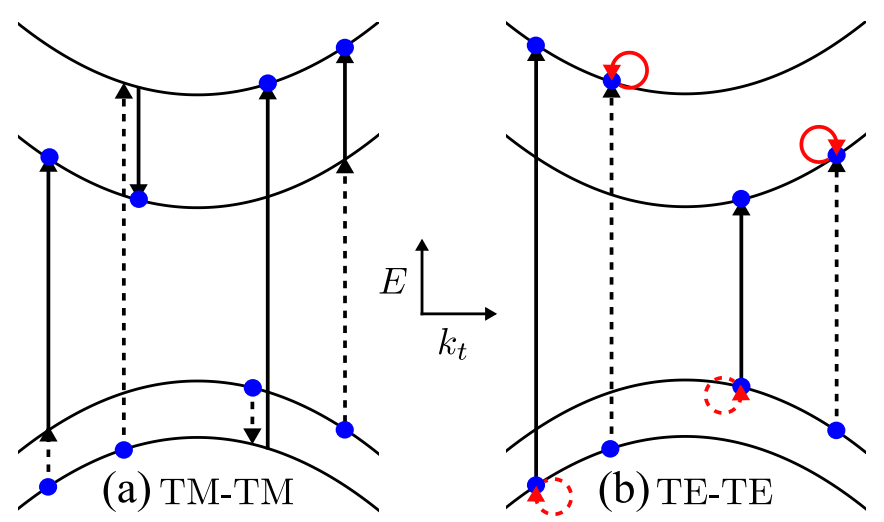

FIG. 3. A diagram of the possible paths for TM-TM (a) and TETE (b) two-photon transitions. The initial and final states are marked with a blue dot. Dashed lines represent a nonresonant transition, which is the transition leading to a detuning denominator. Black and red lines signify allowed and forbidden transitions, respectively. 


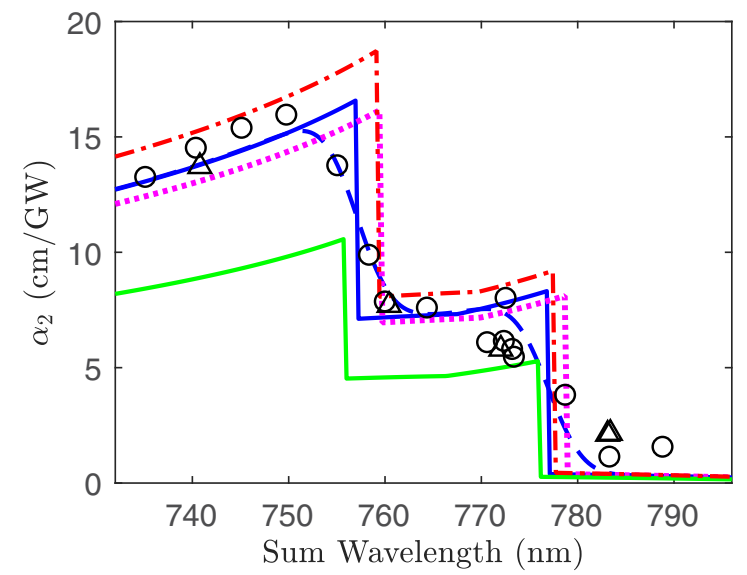

FIG. 4. TM-TM ND-2PA coefficients vs $\lambda_{\text {sum }}=\left(1960^{-1}+\right.$ $\left.\lambda_{1}^{-1}\right)^{-1}(\mathrm{~nm})$. Black circles: measured coefficients with total pulse energy $E_{2}=3.5 \mathrm{pJ}$. Black triangles: measured coefficients with $E_{2}=4.9 \mathrm{pJ}$. Red dash-dotted line: theory with eight-band basis. Solid blue line: theory with 14-band basis. Dashed blue line: TM-TM theory convolved with $4 \mathrm{~nm}$ Gaussian to approximate bandwidth effects. Magenta dotted line: 14-band model without adjustments to well width and barriers. Solid green line: 14-band calculation with $E_{p}=25 \mathrm{eV}$.

function to

$$
\begin{aligned}
f_{2}\left(x_{1} ; x_{2}\right)= & \frac{4}{x_{1} x_{2}^{2}}\left(\frac{1}{x_{1}}+\frac{1}{x_{2}}\right)^{2} \sum_{c \alpha, v}\left\langle\left|M_{c \alpha, v}^{y} \sin \phi\right|^{2}\right\rangle_{\phi} \\
& \times\left[x_{1}+x_{2}-\epsilon_{c v}(0)\right] \Theta\left[x_{1}+x_{2}-\epsilon_{c v}(0)\right] .
\end{aligned}
$$

The term linear in $x_{1}+x_{2}$ grows from zero at the 2PA edge, meaning TE-TE 2PA dispersion lacks the discontinuities seen in TM-TM 2PA curves.

The $\phi$ integration has been reduced to the average over a single term denoted with angular brackets. Performing the integration for each pair of bands gives

$$
\begin{aligned}
& \left\langle\left|M_{\mathrm{c} \alpha, l \alpha}^{y} \sin \phi\right|^{2}\right\rangle=\frac{1}{8}(1-\cos 2 \theta), \\
& \left\langle\left|M_{\mathrm{c} \alpha, l \beta}^{y} \sin \phi\right|^{2}\right\rangle=\frac{1}{96}(5+3 \cos 2 \theta), \\
& \left\langle\left|M_{\mathrm{c} \alpha, h \beta}^{y} \sin \phi\right|^{2}\right\rangle=\frac{1}{96}(17-9 \cos 2 \theta) .
\end{aligned}
$$

Note that if we chose $x$ polarized light, we would need to use the $x$ components of the interband matrix elements and take $\hat{\mathbf{e}} \cdot \mathbf{k}_{t}=\cos \phi$. The result is that the integration over $\phi$ yields identical values to $y$ polarized light. This equivalence is consistent with the fact that physical measurements must have the azimuthal symmetry of the isotropic bands.

\section{RESULTS}

Normalized transmission versus pump-probe delay was measured as described in Sec. III, and coupled nonlinear Schrödinger equations were used to fit the curves to ND-2PA coefficients. The exact procedure is detailed in Appendix B, along with all necessary approximations and empirical adjustments.

TM-TM measurement results are plotted versus sum wavelength in Fig. 4 alongside theoretical predictions. Sum wavelength is defined by $1 / \lambda_{\text {sum }}=1 / \lambda_{1}+1 / \lambda_{2}$, where the pump
TABLE III. Quantum well subband energies (in meV) at $\kappa=0$ for $7.84 \mathrm{~nm} \mathrm{GaAs}$ quantum wells with $\mathrm{Al}_{0.328} \mathrm{Ga}_{0.672}$ As barriers.

\begin{tabular}{cccc}
\hline \hline & $c$ & lh & hh \\
\hline 1 & 1475.9 & -33.5 & -11.5 \\
2 & 1604.9 & -120.3 & -45.4 \\
3 & & & -98.9 \\
\hline \hline
\end{tabular}

wavelength $\lambda_{2}$ is fixed at $1960 \mathrm{~nm}$. Excitations of light hole states bring about discontinuities in the spectrum, with the $l 1 \rightarrow c 2$ and $l 2 \rightarrow c 1$ transitions accounting for the shorter and longer wavelength steps, respectively. In contrast with light hole contributions, heavy hole signals exhibit a gradual increase due to the interband $y$ matrix element's $\sin \theta$ (forbidden) dependence. Each transition's onset is found by subtracting the subband energies in Table III using the selection rule $m-n= \pm 1$.

Expanding states in the 8-band basis with $E_{p}=28.9 \mathrm{eV}$ [34], our predicted curve matched the data apart from a 3-nm wavelength shift. This offset is mitigated by using the 14-band model with $E_{p}^{\prime}=6 \mathrm{eV}$ [34]. In both cases, we assumed there were small growth errors such that the real material consisted of 7.84-nm wells with $\mathrm{Al}_{0.328} \mathrm{Ga}_{0.672} \mathrm{As}$ barriers. The effect of this modification is revealed by comparing the blue (solid) and magenta (dotted) curves of Fig. 4. Other sources for this wavelength inaccuracy could be OSA miscalibration or the use of imprecise band-gap values in simulations, but these assumptions lead to curves nearly identical to the ones shown.

Interestingly, calculated 2PA coefficients vary significantly with the value chosen for the Kane energy. This sensitivity is apparent when comparing the 14-band theoretical curve with another that has $E_{p}=25 \mathrm{eV}$ and $E_{p}^{\prime}=6 \mathrm{eV}$ [34]. By the arguments of Sec. IV A, under these conditions we require $m_{0} / \mathscr{M}_{c c}^{z z}=3.0$ so that Eq. (15) reduces to the bulk effective mass for GaAs. For comparison, $m_{0} / \mathscr{M}_{c c}^{z z}=-2.2$ when $E_{p}=$ $28.9 \mathrm{eV}$. This modification reduces the intersubband matrix element according to Eq. (17).

Figure 5 shows the TE-TE results compared with 14-band calculations. The ND-2PA edge is energetically lower than in TM-TM because it first occurs for $h 1 \rightarrow c 1$ transitions, leading to large anisotropy below the $l 1 \rightarrow c 2$ TM-TM transition energy. The theoretical curve, which is smooth with a kink at $750 \mu \mathrm{m}$ from $h 2 \rightarrow c 2$ transitions, shows this anisotropy. However, our unscaled calculations differ from the measurements by over a factor of 4 and show some dissimilarity in shape. We provide reasons for these differences in the following section.

\section{DISCUSSION}

The TM-TM theory matches the data without any nonphysical scaling parameter; this excellent agreement is likely due to the dominance of allowed transitions. The parabolic band approximation works well because important features in the 2PA spectrum occur at small $\mathbf{k}_{t}$, where the parabolic band approximation introduces little error. Calculations show that the ND-2PA coefficients measured here are enhanced by a factor of 1.5 over degenerate $2 \mathrm{PA}$. 


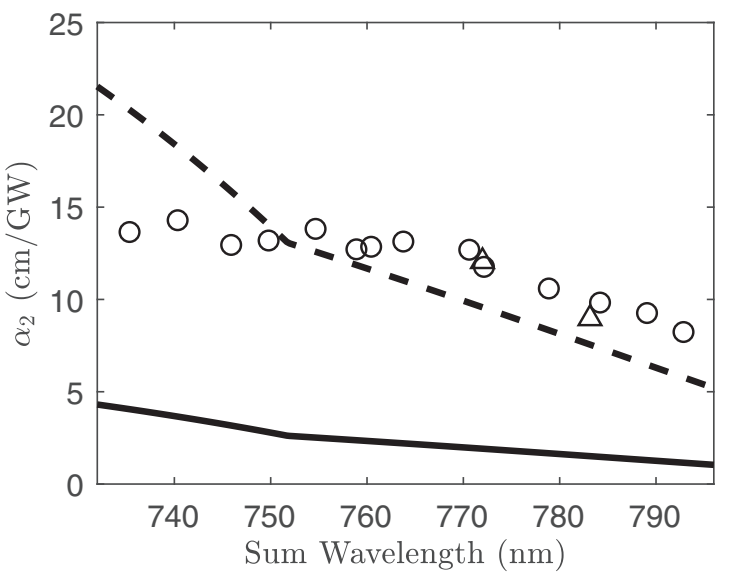

FIG. 5. Comparison between theory and experiment for TETE polarization. Black circle: TE-TE ND-2PA coefficients at $E=$ 4.2 pJ. Black diamond: TE-TE ND-2PA coefficients with $E=$ $6.0 \mathrm{pJ}$. Black line: TE-TE 2PA theory. Black dotted line: TE-TE 2PA theory multiplied by 4 .

We do not notice any bound excitonic response, which may be attributed to temperature effects. Continuum exciton enhancement is also not evident. Reference [14] concludes that this contribution is absent in Ref. [15] due to low sample quality and large exciton spatial extent. We suspect the same reasons apply here, with further reductions possibly occurring due to loss of $2 \mathrm{D}$ character from interactions between many closely spaced wells.

In contrast to TM-TM polarizations, the parabolic band approximation introduces significant errors in TE-TE ND2PA coefficients. By ignoring unit-cell intermixing, we underestimate the $\mathbf{k}_{t}$-dependent scaling of forbidden transitions that are necessary in TE-TE pathways. We also determine that it is insufficient to examine only self-transitions as the forbidden step; we must also consider intersubband transitions and those between different hole types. Away from the band edge, light-hole to heavy-hole transitions were shown to be non-negligible for 2PA in bulk semiconductors [24]. TE-TE coefficients could be more accurately calculated by numerically computing the highly nonparabolic band dispersion as in Ref. [35]. Equation (6) would then give matrix elements throughout the Brillouin zone, which are used to find 2PA coefficients according to Eq. (8).

The sensitivity of the 2PA coefficient to Kane energy and effective masses indicates that pump probe spectroscopy of quantum wells may be an effective method for determining basic material parameters. Our results, while not precise enough to justify a definitive declaration, seem to support the idea that the Kane energy is closer to $28.9 \mathrm{eV}$ than lower values that have been reported (see Appendix A). Furthermore, Hübner et al. [36] and others have shown evidence that the Kane energy is dependent on temperature. With better spectral resolution and careful experimental setup, the temperature dependence of $E_{p}$ could be reflected both in a magnitude change of the normalized transmission signal and a shift of the ND-2PA edge as effective mass is changed [see Eq. (A4)].

With this experimental support for our model, we can reexamine ND-2PA for extremely nondegenerate photon pairs

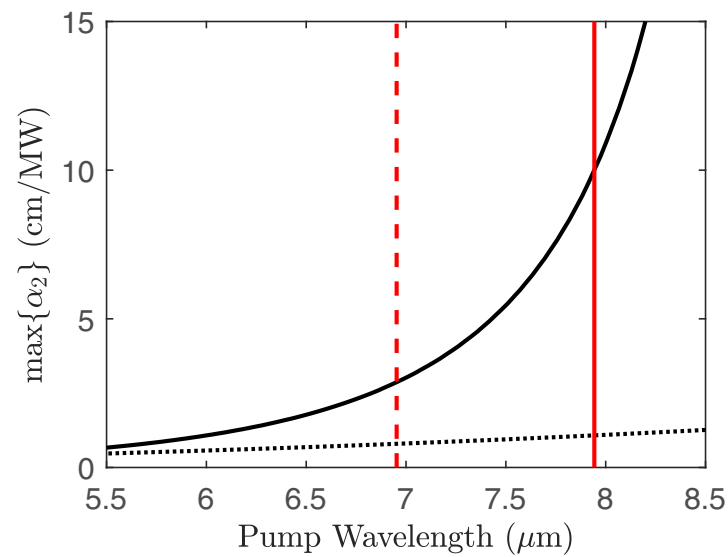

FIG. 6. Maximum TM-TM ND-2PA coefficient (in $\mathrm{cm} / \mathrm{MW}$ ) vs pump wavelength. The dashed red line denotes the point where maximum 2PA occurs for a probe wavelength within $25 \mathrm{meV}$ of the $h 1 \rightarrow c 1$ band gap. The solid red line is the same for the $l 1 \rightarrow c 1$ gap. The dotted black line shows the END-2PA coefficients of bulk GaAs for a probe just below the band gap [37].

$[17,18,37]$. Figure 6 shows that the calculated TM-TM ND2PA coefficients rapidly increase as the pump wavelength nears the $c 1 \rightarrow c 2$ resonance at $9.55 \mu \mathrm{m}$. We can compare this enhancement to that seen in bulk GaAs (dotted line) arising from a zero resonance. The red vertical lines denote points where the probe energy for maximum 2PA lies within $k T=25 \mathrm{meV}$ of the forbidden (dashed, $h 1 \rightarrow c 1$ ) and allowed (solid, $l 1 \rightarrow c 1$ ) linear absorption edges. The offset value is chosen so that we can roughly assume negligible impurity state absorption, but these edges will shift depending on material quality and temperature. For $\lambda_{2}=7.5 \mu \mathrm{m}$, we see that $\max \left\{\alpha_{2}\right\}=5.7 \mathrm{~cm} / \mathrm{MW}$-an enhancement of $\sim 360 \times$ over the slightly nondegenerate case studied here, and a considerably larger ND-2PA coefficient than any we have measured in bulk semiconductors $(\sim 1 \mathrm{~cm} / \mathrm{MW})$ [37]. This enhancement suggests the possibility of extremely sensitive gated detection as in Ref. [1], with even further increases in photogenerated carrier density due to long pulse interactions within a waveguide.

\section{ACKNOWLEDGMENTS}

Funding for N.C., D.H., and E.V.S. was provided by National Science Foundation Grant No. DMR-1609895 and the Army Research Laboratory ( Grant No. W911NF-15-2-0090). This work was performed, in part, at the Center for Integrated Nanotechnologies, an Office of Science User Facility operated for the U.S. Department of Energy (DOE) Office of Science by Los Alamos National Laboratory (Contract No. 89233218CNA000001) and Sandia National Laboratories (Contract No. DE-NA-0003525). J.W. and S.P.G. acknowledge the support from the Fonds de la Recherche Fondamentalei Collective (FRFC) (Grant No. PDR.T.1084.15).

\section{APPENDIX A: SIMULATION DETAILS}

Since we are not working with an idealized structure, we must employ some numerical techniques to model our 
systems. In the first subsection of this Appendix, we calculate energy levels and envelopes for states in the finite quantum well. In the second, we find the optical mode structure and dispersion characteristics of the waveguide.

\section{Material simulations}

We first give the band gap of GaAs and the compositiondependent band gap of AlGaAs in order to determine the confining potential imposed by the AlGaAs barriers. Then we provide values for interband couplings and effective masses, followed by a brief summary of the calculation results. Each value is taken from the literature, making adjustments as needed.

The temperature-dependent band gap of GaAs is given by the Varshni relation [38]

$$
E_{g}=1.519-\alpha \frac{T^{2}}{T+\beta} \quad(\mathrm{eV})
$$

with $\alpha=8.95 \times 10^{-4} \mathrm{eV} / \mathrm{K}$ and $\beta=538 \mathrm{~K}[39,40]$. The higher conduction-band energies in the 14-band model are taken to be $E_{g}^{\prime}=4.63 \mathrm{eV}$ and $E_{s^{\prime}}=4.44 \mathrm{eV}$ [41].

The potential barriers imposed by the quantum well layer structure come from the empirical expression for total band offset between $\mathrm{GaAs}$ and $\mathrm{Al}_{x} \mathrm{Ga}_{1-x}$ As [39]:

$$
\Delta E_{g}=1.395 x \quad(x \leqslant 0.41) .
$$

The ratio $Q=\Delta E_{v} / \Delta E_{g}=0.33$ [40] at an $\mathrm{Al}_{x} \mathrm{Ga}_{1-x}$ As interface, simplifying conduction and valence offsets to

$$
\begin{aligned}
& \Delta E_{c}=V_{c}=0.963 x \quad(\mathrm{eV}), \\
& \Delta E_{v}=V_{h}=-0.432 x \quad(\mathrm{eV}) .
\end{aligned}
$$

All hole types are presumed to have offset $V_{h}$ and all conduction bands are taken to have offset $V_{c}$.

Literature values for the Kane energy of GaAs vary between $E_{p}=22.9 \mathrm{eV}$ [42], $25.7 \mathrm{eV}$ [43], $27.86 \mathrm{eV}$ [44], $28.8 \mathrm{eV}$ [45], and $28.9 \mathrm{eV}$ [34]. We choose $E_{p}=28.9 \mathrm{eV}$, and take the inter-conduction band coupling strength as $E_{p}^{\prime}=6 \mathrm{eV}$ [34].

The conduction-band effective mass of GaAs is $m_{c}^{z}=m_{c}^{t}=$ $0.0635 m_{0}$ [40] at room temperature. Light-hole masses are anisotropic with $m_{l}^{z}=-0.082 m_{0}$ [46] as determined from cyclotron resonance at $77 \mathrm{~K}$. This mass is adjusted to its room-temperature value by

$$
\frac{1}{m_{l, 295 \mathrm{~K}}}=\frac{1}{m_{l, 77 \mathrm{~K}}}+\frac{2 E_{p}}{3}\left(\frac{1}{E_{g, 295 \mathrm{~K}}}-\frac{1}{E_{g, 77 \mathrm{~K}}}\right),
$$

where temperature-dependent band gaps are taken from Eq. (A1). This relation comes from subtracting the Eq. (14) expressions for 295 and $77 \mathrm{~K}$. The final outcome is that $m_{l, 295 \mathrm{~K}}^{z}=-0.077 m_{0}$.

The energy-dependent effective mass is calculated in the eight-band model using Eq. (14) with spin-orbit splitting of $\Delta=0.341 \mathrm{eV}$ [47].

The heavy-hole effective mass $m_{h}(z)$ is [20]

$$
m_{h}^{z}(z)=\frac{m_{0}}{\gamma_{1}[x(z)]-2 \gamma_{2}[x(z)]},
$$

where $x(z)$ is the AlGaAs composition at position $z$. The symbol $\gamma(x)$ is the six-band Luttinger parameter linearly
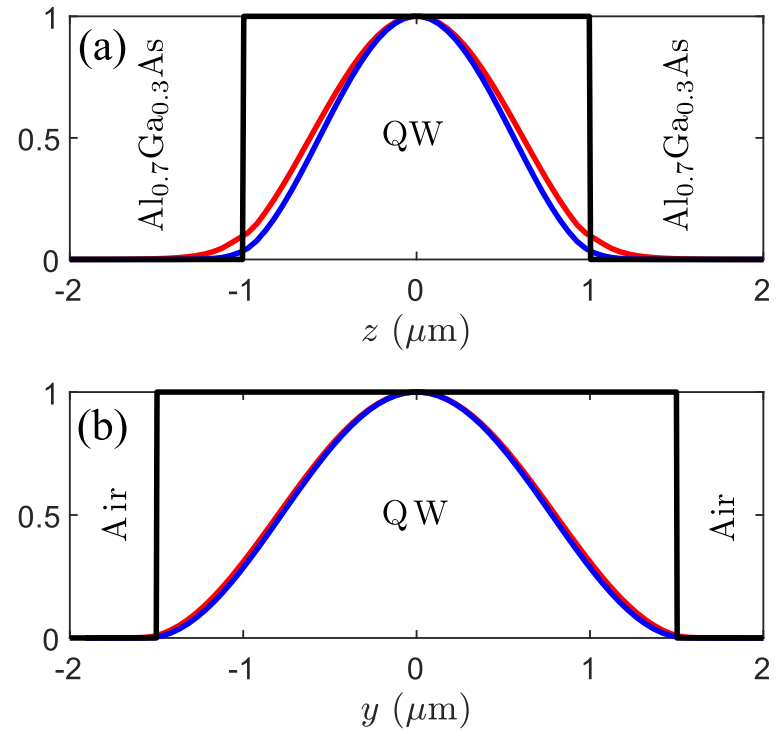

FIG. 7. Normalized pump (red) and probe (blue) TM mode profiles in the $z$ (a) and $y$ (b) directions for the structure shown in Fig. 1.

interpolated between the values for GaAs and AlAs [40]:

$$
\begin{aligned}
& \gamma_{1}(x)=6.98-(3.76-6.98) x, \\
& \gamma_{2}(x)=2.06-(0.82-2.06) x .
\end{aligned}
$$

Transverse hole effective masses are also taken from the Luttinger parameters as [20]

$$
\begin{aligned}
m_{h}^{t} & =\frac{m_{0}}{\gamma_{1}(0)+\gamma_{2}(0)}, \\
m_{l}^{t} & =\frac{m_{0}}{\gamma_{1}(0)-\gamma_{2}(0)} .
\end{aligned}
$$

With all material parameters known, the shooting method [48] is used to solve Eq. (13). The procedure yields the energy level (see Table III) and dominant wave-function envelope for each state. Note that the material widths and compositions are slightly altered as explained in Sec. V.

\section{Waveguide modes}

Refractive index values for the various AlGaAs compositions used were calculated by Adachi's formulas [49]. The index of the quantum well active region was estimated to be the spatial average of the well $(w)$ and barrier $(b)$ permittivities,

$$
n^{2}(x, \lambda)=\frac{L_{w} n_{w}(x, \lambda)^{2}+L_{b} n_{b}(x, \lambda)^{2}}{L_{w}+L_{b}} .
$$

Using these indices, electromagnetic mode profiles and dispersion curves were calculated from the finite difference method with Lumerical MODE Solutions [50]. The mode shapes are shown in Fig. 7. The third-order mode areas $a_{i}$ and overlap $\eta_{i j}$ were calculated in the usual way [51]:

$$
\begin{gathered}
a_{i}=\frac{\left[\iint\left|F_{i}(y, z)\right|^{2} d y d z\right]^{2}}{\iint\left|F_{i}(y, z)\right|^{4} d y d z}, \\
\eta_{i j}=\frac{\iint\left|F_{i}(y, z)\right|^{2}\left|F_{j}(y, z)\right|^{2} d y d z}{\left[\iint\left|F_{i}(y, z)\right|^{4} d y d z\right]^{1 / 2}\left[\iint\left|F_{j}(y, z)\right|^{4} d y d z\right]^{1 / 2}},
\end{gathered}
$$


where $F_{i}(y, z)$ is the electric-field profile of mode $i$. The calculated TM (TE) mode area at $1960 \mathrm{~nm}$ was found to be 3.56 (3.48) $\mu \mathrm{m}^{2}$, and the TM (TE) probe mode areas range from $3.14(3.07) \mu \mathrm{m}^{2}$ at $1150 \mathrm{~nm}$ to $3.21(3.17) \mu \mathrm{m}^{2}$ at $1350 \mathrm{~nm}$. The mode overlap at 1176 and $1960 \mathrm{~nm}$ was $\eta_{12}=0.9967$, so they are treated as unity. Due to tight optical confinement within the active region, propagation is well approximated by taking modes to travel through a material entirely made up of the quantum wells.

We numerically differentiated the refractive index curves to find group velocities and second-order dispersions. The largest GVM of $\rho=\Delta \beta=860 \mathrm{fs} / \mathrm{mm}$ occurred when $\lambda_{1}=$ $1176 \mathrm{~nm}$ and $\lambda_{2}=1960 \mathrm{~nm}$, with the pump traveling faster than the probe. Around this value, $150 \mathrm{fs}$ pulses walk off from each other on the length scale of $350 \mu \mathrm{m}$. The largest dispersion coefficient of $\beta_{2}=1841 \mathrm{fs}^{2} / \mathrm{mm}$ occurs at the same probe wavelength, while the pump dispersion is $\beta_{2}^{(2)}=$ $741 \mathrm{fs}^{2} / \mathrm{mm}$ at $1960 \mathrm{~nm}$. These values were used in the simulations of Appendix B.

\section{APPENDIX B: NONLINEAR PROPAGATION AND DATA FITTING}

We modeled pulse propagation by the coupled nonlinear Schrodinger equations [52]

$$
\begin{gathered}
\left(\frac{\partial}{\partial x}+\beta_{1}^{(1)} \frac{\partial}{\partial t}+i \frac{\beta_{2}^{(1)}}{2} \frac{\partial^{2}}{\partial t^{2}}+\frac{\sigma_{1}}{2}\right) A_{1} \\
=i\left(\gamma_{11}\left|A_{1}\right|^{2}+2 \gamma_{12}\left|A_{2}\right|^{2}\right) A_{1}, \\
\left(\frac{\partial}{\partial x}+\beta_{1}^{(2)} \frac{\partial}{\partial t}+i \frac{\beta_{2}^{(2)}}{2} \frac{\partial^{2}}{\partial t^{2}}+\frac{\sigma_{2}}{2}\right) A_{2} \\
=i\left(\gamma_{22}\left|A_{2}\right|^{2}+2 \gamma_{21}\left|A_{1}\right|^{2}\right) A_{2} .
\end{gathered}
$$

$\left|A_{l}\right|^{2}$ is the instantaneous power, $\beta_{1}^{(l)}$ and $\beta_{2}^{(l)}$ are the first- and second-order dispersion, and $\sigma_{l}$ is the loss. We neglect freecarrier contributions to the nonlinear propagation because the pulses have sufficiently low average power such that excited carrier density is negligible. The nonlinear parameter $\gamma_{i j}$ is written in terms of the mode areas and overlap as [51]

$$
\gamma_{i j}=\frac{\omega_{i}}{c} \frac{n_{2}\left(\omega_{i} ; \omega_{j}\right) \eta_{i j}}{\sqrt{a_{i} a_{j}}}+i \frac{\alpha_{2}\left(\omega_{i} ; \omega_{j}\right) \eta_{i j}}{2 \sqrt{a_{i} a_{j}}} .
$$

We set $\operatorname{Im}\left\{\gamma_{22}\right\}=0$ because the pump wavelength is below the D-2PA edge, and the $\gamma_{i 1}$ are ignored because the probe power is low. All nonlinear refraction effects from $\operatorname{Re}\left\{\gamma_{i j}\right\}$ are ignored, which is justified in the following subsection.

\section{Raw data and analysis}

Figure 8 shows a normalized transmission signal generated as described in Sec. III. A delay of zero indicates that the pump and probe arrive at the front facet at the same time, and a negative delay means the probe arrives before the pump. The curve is temporally wider than the input pulses because the faster moving pump overtakes the probe within the sample for delays up to about -2.3 ps. Transmission curves in high GVM experiments usually exhibit a tabletop shape like those in Ref. [37]. Instead, we see a decay in the nonlinear

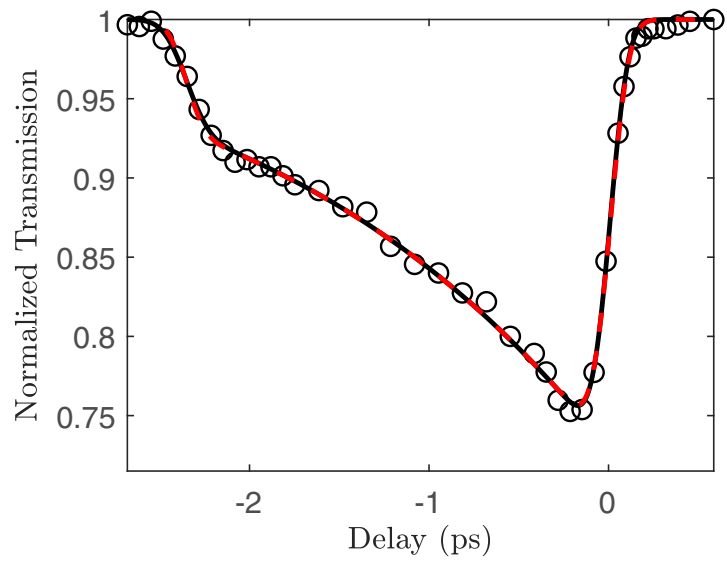

FIG. 8. Normalized transmission vs delay at $\lambda_{2}=1960 \mathrm{~nm}$ and $\lambda_{1}=1246 \mathrm{~nm}$. The results are compared to calculations using the split-step Fourier method (black line) and analytical expression of Eq. (B4) (red dashed line). The parameters used were $\operatorname{Im}\left\{\gamma_{12}\right\}=$ $12.9 \mathrm{fs} /(\mathrm{mm} \mathrm{pJ}), E_{2}=4.8 \mathrm{pJ}, \rho=654 \mathrm{fs} / \mathrm{mm}, L=3.6 \mathrm{~mm}, \tau_{1}=$ $121 \mathrm{fs}, \tau_{2}=154 \mathrm{fs}, \sigma_{2}=0.4 \mathrm{~mm}^{-1}$. The total width of the signal is $\tau=\rho L=2.35 \mathrm{ps}$.

transmission magnitude as delay becomes more negative. This indicates that loss in pump irradiance leads to a smaller signal as the pulses meet after propagating farther through the sample. If these losses were caused by an interband absorption process, there would be a constant signal at positive delay due to excited carrier interactions. Instead, we attribute this decay to high scattering from roughness of the etched sidewall.

The shape of Fig. 8 is explained by the analytical expression for normalized transmission,

$$
\begin{aligned}
\Delta T= & \exp \left\{2 \frac{E_{2} \operatorname{Im}\left\{\gamma_{12}\right\}}{\rho} \exp \left[\frac{\sigma_{2} \tau}{\rho}+\left(\frac{\sigma_{2} \tau_{x}}{2 \rho}\right)^{2}\right]\right. \\
& \left.\times\left[\operatorname{erf}\left(\frac{\tau}{\tau_{x}}+\frac{\sigma_{2} \tau_{x}}{2 \rho}\right)-\operatorname{erf}\left(\frac{\tau+\rho L}{\tau_{x}}+\frac{\sigma_{2} \tau_{x}}{2 \rho}\right)\right]\right\},
\end{aligned}
$$

which is derived in detail in Appendix G. Here, $L$ is the length of the sample and $E_{2}$ is the total energy of the pump pulse. The value $\tau_{x}=\left(\tau_{1}^{2}+\tau_{2}^{2}\right)^{1 / 2}$ is the pure cross correlation width between pulse durations $\tau_{j}$, and $\rho=\beta_{1}^{(2)}-\beta_{1}^{(1)}$ is the GVM parameter. Equation (B4) was derived by ignoring second-order dispersion. Despite this approximation, we see by comparing the curves in Fig. 8 that the equation yields nearly identical results to those found by split-step Fourier integration of Eqs. (B1) and (B2) with nonzero $\beta_{2}$.

The insensitivity to $\beta_{2}$ is related to an overall pulse width insensitivity of the normalized transmission. Qualitatively, the increase in pump irradiance at fixed energy due to decrease in pulse width is compensated by a decrease in interaction length as the pulses walk through each other. In fact, with $\sigma_{2}=0$, the maximum signal of Eq. (B4) is completely independent of pulse widths. Since the linear and nonlinear pulse broadening should be negligible over the $\sim 300-\mu \mathrm{m}$ walkoff length, this independence would be true without ignoring $\operatorname{Re}\left\{\gamma_{i j}\right\}$ and $\beta_{2}^{(l)}$. In our case, altering the pulse width only causes a slight signal 
reduction due to a delay shift in the curve moving the peak back to a point where more pump losses have occurred. This only gives an error of around $8 \%$ when $\tau_{x}$ is underestimated by a factor of 2 . We use this insensitivity to justify ignoring nonlinear refraction in Eqs. (B1) and (B2).

\section{Data fitting procedure}

For TM-TM (TE-TE) sum wavelengths of 743, 762, 772, $784 \mathrm{~nm}(743,762,780 \mathrm{~nm})$ the transmission curves were fit with $\operatorname{Im}\left\{\gamma_{12}\right\}, \sigma_{2}, \rho$, and $\tau_{1}$ as free parameters. Averaging the resulting loss coefficents gives $\sigma_{2, \mathrm{TM}}=0.46 \mathrm{~mm}^{-1}$ and $\sigma_{2, \mathrm{TE}}=0.56 \mathrm{~mm}^{-1}$. A wavelength shift of $25 \mathrm{~nm}$ was applied to the dispersion curve so the simulated values of $\rho$ more closely match the fits. This adjustment is needed most likely due to the inaccuracy of the spatially averaged approximation to the quantum well index [Eq. (A9)]. The values presented here were held fixed in fitting the rest of the data.

The rest of the data points in Figs. 4 and 5 were found by measuring the rising edge and peak of the normalized transmission. The pump pulse width was taken to be $9 \%$ greater than the bandwidth limit (see Sec. III), and $\gamma_{12}$ and $\tau_{1}$ were free fitting parameters. Once again, the effect of this imperfect knowledge of pulse widths is mitigated by the signal's insensitivity to pulse width. The $\sigma_{2}$ and GVM values were held fixed according to the previous fitting procedure.

\section{APPENDIX C: DERIVATION OF 2PA COEFFICIENTS}

Here we derive the 2PA coefficients for a quantum well. Some steps will be repeated from the brief version in Sec. II; we start again with the second-order transition rate per unit volume,

$$
\begin{aligned}
W= & \frac{2 \pi}{\hbar} \frac{1}{V} \sum_{\mathbf{k}_{t}} \sum_{c v}\left|\frac{H_{c i}^{2} H_{i v}^{1}}{E_{i v}-\hbar \omega_{1}}+\frac{H_{c i}^{1} H_{i v}^{2}}{E_{i v}-\hbar \omega_{2}}\right|^{2} \\
& \times \delta\left(\hbar \omega_{1}+\hbar \omega_{2}-E_{c v}\right),
\end{aligned}
$$

where $H_{m n}=-e /\left(2 m_{0}\right) \mathbf{A}_{0} \cdot \mathbf{p}$ for a harmonic vector potential of magnitude $\mathbf{A}_{0}$. We define a dimensionless matrix element $M=\hbar /\left(m_{0} P\right) \hat{\mathbf{e}} \cdot \mathbf{p}$ so that

$$
H_{m n}=-\frac{e A_{0}}{2 \hbar} P M .
$$

Substituting the normalized matrix element into the transition rate per unit volume leads to

$$
\begin{aligned}
W= & \frac{2 \pi}{\hbar^{5}}\left(\frac{e}{2}\right)^{4} \frac{\left|A_{01} A_{02}\right|^{2}}{V} P^{4} \\
& \times \sum_{c v, \mathbf{k}_{t}}\left|\frac{M_{c i}^{2} M_{i v}^{1}}{E_{i v}-\hbar \omega_{1}}+\frac{M_{c i}^{1} M_{i v}^{2}}{E_{i v}-\hbar \omega_{2}}\right|^{2} \\
& \times \delta\left(\hbar \omega_{1}+\hbar \omega_{2}-E_{c v}\right) .
\end{aligned}
$$

At this point the derivation becomes much cleaner in atomic units where $\hbar=m_{0}=e=1 /\left(4 \pi \epsilon_{0}\right)=1$. Changing units and normalizing all energies to the band gap $E_{g}$ gives

$$
\begin{aligned}
W= & 2 \pi \frac{A_{01}^{2} A_{02}^{2}}{16} \frac{1}{V} \frac{P^{4}}{E_{g}^{3}} \sum_{\mathbf{k}_{t}} \sum_{c v}\left|\frac{M_{c i}^{2} M_{i v}^{1}}{\epsilon_{i v}-x_{1}}+\frac{M_{c i}^{1} M_{i v}^{2}}{\epsilon_{i v}-x_{2}}\right|^{2} \\
& \times \delta\left(x_{1}+x_{2}-\epsilon_{c v}\right) .
\end{aligned}
$$

We now convert the sum to an integral over the first Brillouin zone by

$$
\frac{2 \pi}{V} \sum_{\mathbf{k}_{t}} \rightarrow \frac{2 \pi}{V} \int_{B Z} \frac{L_{x} L_{y}}{(2 \pi)^{2}} d \mathbf{k}_{t} .
$$

Since $V_{c}=L_{x} L_{y} L_{z}$ is the volume of the crystal, we find (after changing to polar coordinates)

$$
\frac{2 \pi}{V_{c}} \sum_{\mathbf{k}_{t}} \rightarrow \frac{1}{L_{z}} \frac{1}{2 \pi} \int_{0}^{2 \pi} d \phi \int_{0}^{\text {z.b. }} k d k .
$$

The upper limit of the $k$ integral accounts for the fact that the distance to the zone boundary depends on the angle $\phi$, though this limit has very little practical effect on the results. Noting that $k P$ has units of energy, we can define another normalized parameter $\kappa=k P / E_{g}$. Updating the integration variable to $\kappa$ leads to

$$
W=\frac{A_{01}^{2} A_{02}^{2}}{16} \frac{P^{2}}{E_{g}} f_{2}\left(\frac{\hbar \omega_{1}}{E_{g}} ; \frac{\hbar \omega_{2}}{E_{g}}\right),
$$

with the dimensionless spectral function defined to be

$$
\begin{aligned}
f_{2}\left(x_{1} ; x_{2}\right)= & \sum_{c v} \frac{1}{2 \pi} \int_{0}^{2 \pi} d \phi \int_{0}^{\text {z.b. }} \kappa\left|\sum_{i} \frac{M_{c i}^{2} M_{i v}^{1}}{\epsilon_{i v}-x_{1}}+\frac{M_{c i}^{1} M_{i v}^{2}}{\epsilon_{i v}-x_{2}}\right|^{2} \\
& \times \delta\left(x_{1}+x_{2}-\epsilon_{c v}\right) d \kappa .
\end{aligned}
$$

Now we convert the transition rate to a $2 \mathrm{PA}$ coefficient by

$$
\alpha_{2}\left(x_{1} ; x_{2}\right)=\frac{x_{1} E_{g}}{2 I_{1} I_{2}} W
$$

with the irradiance $I_{l}$ given in atomic units as

$$
I_{l}=\frac{c}{8 \pi} n_{l} A_{0 l}^{2} x_{l}^{2} E_{g}^{2}
$$

Plugging this in and simplifying gives

$$
\alpha_{2}\left(\omega_{1} ; \omega_{2}\right)=K \frac{E_{p}}{n_{1} n_{2} E_{g}^{4} L_{z}} f_{2}\left(\frac{\hbar \omega_{1}}{E_{g}} ; \frac{\hbar \omega_{2}}{E_{g}}\right),
$$

where $K$ is the material-independent parameter

$$
K=\left(\frac{\pi}{c}\right)^{2}=\left(\frac{\pi}{137}\right)^{2} .
$$

We perform the delta function integration on the dimensionless spectral function using the identity

$$
\int f(x) \delta[g(x)] d x=\sum_{x_{0}} f\left(x_{0}\right)\left|\frac{\partial g}{\partial x}\right|_{x_{0}}^{-1},
$$

where $x_{0}$ is a real solution to the equation $g(x)=0$. Finally, we find that

$$
\begin{aligned}
f_{2}= & \sum_{c v} \frac{1}{2 \pi} \int_{0}^{2 \pi} d \phi \sum_{\kappa_{0}} \kappa_{0}\left|\frac{\partial \epsilon_{c v}}{\partial \kappa}\right|_{\kappa_{0}}^{-1} \\
& \times\left|\sum_{i} \frac{M_{c i}^{2} M_{i v}^{1}}{\epsilon_{i v}-x_{1}}+\frac{M_{c i}^{1} M_{i v}^{2}}{\epsilon_{i v}-x_{2}}\right|^{2} .
\end{aligned}
$$


TABLE IV. Table of zone-center wave functions and their corresponding energies. The top half shows $\alpha$ spin states and the bottom contains $\beta$ states.

\begin{tabular}{cl}
\hline \hline$E$ & $u_{j^{0}}(\mathbf{r})$ \\
\hline$E_{g}$ & $u_{c \alpha}:|i S \downarrow\rangle$ \\
0 & $u_{h \alpha}:-\sqrt{1 / 2}|(X+i Y) \uparrow\rangle$ \\
0 & $u_{l \alpha}: \sqrt{1 / 6}|(X-i Y) \uparrow\rangle+\sqrt{2 / 3}|Z \downarrow\rangle$ \\
$-\Delta$ & $u_{s \alpha}: \sqrt{1 / 3}|(X-i Y) \uparrow\rangle-\sqrt{1 / 3}|Z \downarrow\rangle$ \\
$E_{g}^{\prime}$ & $u_{h^{\prime} \alpha}:-\sqrt{1 / 2}\left|\left(X^{c}+i Y^{c}\right) \uparrow\right\rangle$ \\
$E_{g}^{\prime}$ & $u_{l^{\prime} \alpha}: \sqrt{1 / 6}\left|\left(X^{c}-i Y^{c}\right) \uparrow\right\rangle+\sqrt{2 / 3}\left|Z^{c} \downarrow\right\rangle$ \\
$E_{s^{\prime}}$ & $u_{s^{\prime} \alpha}: \sqrt{1 / 3}|(X-i Y) \uparrow\rangle-\sqrt{1 / 3}\left|Z^{c} \downarrow\right\rangle$ \\
$E_{g}$ & $u_{c \beta}:|i S \uparrow\rangle$ \\
0 & $u_{h \beta}: \sqrt{1 / 2}|(X-i Y) \downarrow\rangle$ \\
0 & $u_{l \beta}:-\sqrt{1 / 6}|(X+i Y) \downarrow\rangle+\sqrt{2 / 3}|Z \uparrow\rangle$ \\
$-\Delta$ & $u_{s \beta}: \sqrt{1 / 3}|(X+i Y) \downarrow\rangle+\sqrt{1 / 3}|Z \uparrow\rangle$ \\
$E_{g}^{\prime}$ & $u_{h^{\prime} \beta}: \sqrt{1 / 2}\left|\left(X^{c}-i Y^{c}\right) \downarrow\right\rangle$ \\
$E_{g}^{\prime}$ & $u_{l^{\prime} \beta}:-\sqrt{1 / 6}\left|\left(X^{c}+i Y^{c}\right) \downarrow\right\rangle+\sqrt{2 / 3}\left|Z^{c} \uparrow\right\rangle$ \\
$E_{s^{\prime}}$ & $u_{s^{\prime} \beta}: \sqrt{1 / 3}\left|\left(X^{c}+i Y^{c}\right) \downarrow\right\rangle+\sqrt{1 / 3}\left|Z^{c} \uparrow\right\rangle$ \\
\hline \hline
\end{tabular}

\section{APPENDIX D: KANE BAND STRUCTURE}

Kane [53] developed a band-structure model for bulk zinc-blende materials using a $\mathbf{k} \cdot \mathbf{p}$ formalism including spinorbit interaction. The unit-cell basis consisted of two spindegenerate $S$-like functions $|S \uparrow\rangle$ and $|S \downarrow\rangle$ with energy $E_{g}$ and six degenerate $P$-like functions $|X \uparrow\rangle,|X \downarrow\rangle,|Y \uparrow\rangle$, $|Y \downarrow\rangle,|Z \uparrow\rangle$, and $|Z \downarrow\rangle$ with $E=0$. By symmetry of the zinc-blende crystal, all nonzero momentum matrix elements are given by [54]

$$
P=\frac{\hbar}{m_{0}}\left\langle i S\left|p_{x}\right| X\right\rangle=\frac{\hbar}{m_{0}}\left\langle i S\left|p_{y}\right| Y\right\rangle=\frac{\hbar}{m_{0}}\left\langle i S\left|p_{z}\right| Z\right\rangle .
$$

The wave vector in bulk materials is not restricted to two dimensions as in quantum wells. In Ref. [53], the k · p Hamiltonian is diagonalized in a rotated coordinate system for which $\mathbf{k}=k \hat{\mathbf{z}}$. This coordinate transformation is represented as a three-dimensional rotation matrix since $X, Y$ and $Z$ transform as the components of a vector [54]. Finally, the $k$-dependent eigenstates are found to be

$$
\begin{aligned}
& u_{h \alpha}=-\sqrt{1 / 2}|(X+i Y) \uparrow\rangle^{\prime}, \\
& u_{h \beta}=\sqrt{1 / 2}|(X-i Y) \downarrow\rangle^{\prime}, \\
& u_{j \alpha}=a_{j}|i S \downarrow\rangle^{\prime}+b_{j} / \sqrt{2}|(X-i Y) \uparrow\rangle^{\prime}+c_{j}|Z \downarrow\rangle^{\prime}, \\
& u_{j \beta}=a_{j}|i S \uparrow\rangle^{\prime}+b_{j} / \sqrt{2}|-(X+i Y) \downarrow\rangle^{\prime}+c_{j}|Z \uparrow\rangle^{\prime} .
\end{aligned}
$$

The heavy-hole bands $(h)$ are uncoupled while the conduction, light-hole, and split off bands-denoted by index $j-$ intermix. The primed kets indicate rotated basis functions.

The zone center $(\mathbf{k}=0)$ unit-cell functions are listed in Table IV. Taking the first four $\alpha$ and $\beta$ states gives the eight-band Kane basis described above. The more complete 14-band model includes conduction bands at energy $E_{g}^{\prime}$ and $E_{s^{\prime}}=E_{g}^{\prime}-\Delta^{\prime}$, with $\Delta^{\prime}$ representing spin-orbit splitting in the conduction bands. These two sets are used as bases for the envelope expansion in Eq. (12).

\section{Interband matrix elements}

Comparing the $u_{j \mathbf{k}}(\mathbf{r})$ of Eq. (D2) to the zone center $u_{j 0}(\mathbf{r})$ values in Table IV, we note they differ by the expansion coefficients as well as a rotation of basis functions. As in Ref. [33], we assume quantum well transitions are adequately described by using the zone-center expansion coefficient while applying the basis rotation. For example, $\left\langle u_{l \alpha}\left|p_{z}\right| u_{c \alpha}\right\rangle^{\prime}=\left\langle i S\left|p_{z}\right| \sqrt{2 / 3}(Z \cos \theta)\right\rangle$, which evaluates to the value given in Table I after application of Eqs. (D1) and (20).

\section{APPENDIX E: ENVELOPE FUNCTIONS AND EFFECTIVE MASS}

Here we explain the origins of energy-dependent effective masses [Eqs. (14) and (15)] and the envelope Schrödinger equation [Eq. (13)]. What follows is very similar to the derivations in Ref. [27], but we include the details so we can extend the arguments to the 14-band case. We begin by expanding a general wave function in the (assumed complete) set of zone-center wave functions,

$$
\psi_{j n}\left(\mathbf{r} ; \mathbf{k}_{t}\right)=e^{i \mathbf{k}_{t} \cdot \mathbf{r}} \sum_{v} \chi_{j n}^{v}\left(z ; \mathbf{k}_{t}\right) u_{v 0}(\mathbf{r}),
$$

where $\chi_{j n}^{\nu}$ is the envelope component corresponding to the basis function $u_{v 0}(\mathbf{r})$. Absent from the expansion in Eq. (E1) are the class B states included perturbatively using Löwdin's method [20,28].

We first use the eight-band basis with only two spindegenerate conduction bands. Plugging Eq. (E1) into the timeindependent Schrödinger equation gives a new $\mathbf{k} \cdot \mathbf{p}$ form. Defining a vector $\chi$ of the eight envelope functions, the result is found to be

$$
\mathscr{D} \chi=E \chi,
$$

where $\mathscr{D}$ is the matrix [27],

$$
\begin{aligned}
\mathscr{D}_{\nu v^{\prime}}= & \left(E_{n^{\prime}}+\frac{\hbar^{2} k_{t}^{2}}{2 m_{0}}+\frac{p_{z}^{2}}{2 m_{0}}+V(z)\right) \delta_{v v^{\prime}} \\
& +\frac{1}{m_{0}} p_{v v^{\prime}}^{z} p_{z}+\frac{1}{2} p_{z} \frac{1}{\mathscr{M}_{v v^{\prime}}^{z z}} p_{z} \\
& +\frac{\hbar}{m_{0}} \mathbf{k}_{t} \cdot \mathbf{p}_{v v^{\prime}}+\frac{\hbar^{2}}{2} \sum_{\alpha, \beta=x, y} k_{\alpha} \frac{1}{\mathscr{M}_{\nu v^{\prime}}^{\alpha \beta}} k_{\beta} \\
& +\frac{\hbar}{2} \sum_{\alpha=x, y} k_{\alpha} \frac{1}{\mathscr{M}_{v v^{\prime}}^{\alpha z}} p_{z}+p_{z} \frac{1}{\mathscr{M}_{v v^{\prime}}^{\alpha z}} k_{\alpha} .
\end{aligned}
$$

The inverse effective-mass terms $1 / \mathscr{M}$ arise from perturbative inclusion of remote bands using Löwdin's method. These band couplings $v$ and $v^{\prime}$ are described by the tensors

$$
\frac{m_{0}}{\mathscr{M}_{\nu v^{\prime}}^{\alpha \beta}}=\sum_{\lambda} \frac{\left\langle\nu\left|p_{\alpha}\right| \lambda\right\rangle\left\langle\lambda\left|p_{\beta}\right| \nu^{\prime}\right\rangle}{E-E_{\lambda}-V_{\lambda}(z)} .
$$

We can choose $\mathbf{k}_{t}=0$ to simplify Eq. (E3) considerably (the last two rows disappear). This step is where we have taken the parabolic band approximation; we must assume the dispersion to be parabolic in $\mathbf{k}_{t}$ because we are only solving explicitly at the band edge. 
For $\alpha$ states we then have

$$
\begin{array}{r}
\left(E_{c}+\frac{p_{z}^{2}}{2}+V_{c}(z)+\frac{1}{2} p_{z} \frac{1}{\mathscr{M}_{c c}^{z z}} p_{z}\right) \chi_{c}+p_{c l}^{z} p_{z} \chi_{l}+p_{c s}^{z} p_{z} \chi_{s}=E \chi_{c}, \\
\left(E_{l}+\frac{p_{z}^{2}}{2}+V_{l}(z)+\frac{1}{2} p_{z} \frac{1}{\mathscr{M}_{l l}^{z z}} p_{z}\right) \chi_{l}+p_{l c}^{z} p_{z} \chi_{c}+\frac{1}{2} p_{z} \frac{1}{\mathscr{M}_{l s}^{z z}} p_{z} \chi_{s}=E \chi_{l}, \\
\left(E_{h}+\frac{p_{z}^{2}}{2}+V_{h}(z)+\frac{1}{2} p_{z} \frac{1}{\mathscr{M}_{h h}^{z z}} p_{z}\right) \chi_{h}=E \chi_{h}, \\
\left(E_{s}+\frac{p_{z}^{2}}{2}+V_{s}(z)+\frac{1}{2} p_{z} \frac{1}{\mathscr{M}_{s s}^{z z}} p_{z}\right) \chi_{s}+p_{s c}^{z} p_{z} \chi_{c}+\frac{1}{2} p_{z} \frac{1}{\mathscr{M}_{s l}^{z z}} p_{z} \chi_{l}=E \chi_{s},
\end{array}
$$

where we dropped the band index to a subscript because there is no longer room for confusion. The equations for $\beta$ envelopes are identical. Note that the heavy-hole envelope is decoupled from the rest of the basis. This allows the immediate reduction to a Schrödinger equation for the single envelope,

$$
\frac{1}{2} p_{z}\left(1+\frac{1}{\mathscr{M}_{h h}^{z z}}\right) \chi_{h}+V_{h}(z) \chi_{h}=\left(E-E_{h}\right) \chi_{h}
$$

which depends only on the remote band coupling.

The rest of the equations involve intrabasis coupling terms. Assuming valence bands have the same offset $V_{l}=V_{h}=V_{s}=V_{v}$, we rearrange the remaining envelope equations and plug in the appropriate $p_{i j}^{z}$ [from Eq. (D1) and Table IV] to find

$$
\begin{aligned}
& {\left[\frac{1}{2} p_{z}\left(1+\frac{1}{\mathscr{M}_{l l}^{c c}}\right) p_{z}+V_{c}(z)\right] \chi_{c}+\sqrt{\frac{2}{3}} P p_{z} \chi_{l}-\sqrt{\frac{1}{3}} P p_{z} \chi_{s}=\left(E-E_{g}\right) \chi_{c},} \\
& {\left[\frac{1}{2} p_{z}\left(1+\frac{1}{\mathscr{M}_{l l}^{z z}}\right) p_{z}+V_{v}(z)\right] \chi_{l}+\sqrt{\frac{2}{3}} P p_{z} \chi_{c}+\frac{1}{2} p_{z} \frac{1}{\mathscr{M}_{l s}^{z z}} p_{z} \chi_{s}=E \chi_{l},} \\
& {\left[\frac{1}{2} p_{z}\left(1+\frac{1}{\mathscr{M}_{s s}^{z z}}\right) p_{z}+V_{v}(z)\right] \chi_{s}-\sqrt{\frac{1}{3}} P p_{z} \chi_{c}+\frac{1}{2} p_{z} \frac{1}{\mathscr{M}_{s l}^{z z}} p_{z} \chi_{l}=(E+\Delta) \chi_{s} .}
\end{aligned}
$$

With the goal of reducing these to single envelope Schrödinger equations as in Eq. (E6), we would like to eliminate all other envelopes in favor of the dominant one. Clearly, the dominant envelope will be the one corresponding to the band's character. We assume slowly varying envelopes so we can neglect terms of order $p_{z}^{2}$ in comparison to first derivatives. Solving for the dominant envelope in each equation (in order) gives

$$
\begin{aligned}
& \chi_{c}=\sqrt{\frac{1}{3}} \frac{P}{E-E_{g}-V_{c}(z)}\left(\sqrt{2} p_{z} \chi_{l}-p_{z} \chi_{s}\right), \\
& \chi_{l}=\sqrt{\frac{2}{3}} \frac{P}{E-V_{v}(z)} p_{z} \chi_{c}, \\
& \chi_{s}=-\sqrt{\frac{1}{3} \frac{P}{E+\Delta-V_{v}(z)}} p_{z} \chi_{c} .
\end{aligned}
$$

Plugging in the expressions for $\chi_{l}$ and $\chi_{s}$ into the $\chi_{c}$ equation of Eq. (E7) reduces it to an expression for a single envelope,

$$
\frac{1}{2} p_{z}\left(1+\frac{1}{\mathscr{M}_{c c}^{z z}}+\frac{2}{3} \frac{E_{p}}{E-V_{v}(z)}+\frac{1}{3} \frac{E_{p}}{E+\Delta-V_{v}(z)}\right) p_{z} \chi_{c}+V_{c}(z) \chi_{c}=\left(E-E_{g}\right) \chi_{c} .
$$

We eliminated $P$ using $E_{p}=2 P^{2}$ (in atomic units). The effective-mass term sandwiched between the momentum operators now has an energy-dependent portion due to coupling within the basis and an energy-independent portion term due to remote bands. Repeating for light hole envelopes, we find

$$
\left[\frac{1}{2} p_{z}\left(1+\frac{1}{\mathscr{M}_{l l}^{z z}}+\frac{2}{3} \frac{E_{p}}{E-E_{g}-V_{c}(z)}\right) p_{z}+V_{v}(z)\right] \chi_{l}+\frac{1}{2} p_{z}\left(\frac{1}{\mathscr{M}_{l s}^{z z}}+\frac{\sqrt{2}}{3} \frac{E_{p}}{E-E_{g}-V_{c}(z)}\right) p_{z} \chi_{s}=E \chi_{l} .
$$


At first glance, this is more complicated than the conductionband solution because it has not reduced to a single envelope equation. However, we can once again plug in the expression for $\chi_{s}$ as a function of $\chi_{c}$. Finally, we substitute for $\chi_{c}$ in the same way yielding terms of minimum order $p_{z}^{4}$. As always we ignore these relative to the second order. The conclusion is that we may ignore the effect of the $\chi_{s}$ envelope so that

$\frac{1}{2} p_{z}\left(1+\frac{1}{\mathscr{M}_{l l}^{z z}}+\frac{2}{3} \frac{E_{p}}{E-E_{g}-V_{c}(z)}\right) p_{z} \chi_{l}+V_{v}(z) \chi_{l}=E \chi_{l}$.

Repeating for $\chi_{s}$ gives

$\frac{1}{2} p_{z}\left(1+\frac{1}{\mathscr{M}_{s s}^{z z}}+\frac{1}{3} \frac{E_{p}}{E-E_{g}-V_{c}(z)}\right) p_{z} \chi_{s}+V_{v}(z) \chi_{s}=E \chi_{s}$.

\section{4-band model}

The 14-band model comes from a relatively trivial extension of the eight-band analysis. The six added conduction bands have the same symmetry as the valence bands, so they are completely decoupled from each other to first order. Consequently, the valence bands derived in the eight-band model are equally valid in the 14-band case (within the approximations applied). The higher conduction bands are expanded as

$$
\begin{gathered}
\left(E_{l^{\prime}}+\frac{p_{z}^{2}}{2}+V_{l^{\prime}}(z)+\frac{1}{2} p_{z} \frac{1}{\mathscr{M}_{l^{\prime} l^{\prime}}^{z z}} p_{z}\right) \chi_{l^{\prime}} \\
+p_{l^{\prime} c}^{z} p_{z} \chi_{c}+\frac{1}{2} p_{z} \frac{1}{\mathscr{M}_{l^{\prime} s^{\prime}}^{z z}} p_{z} \chi_{s^{\prime}}=E \chi_{l^{\prime}}, \\
\left(E_{s^{\prime}}+\frac{p_{z}^{2}}{2}+V_{s^{\prime}}(z)+\frac{1}{2} p_{z} \frac{1}{\mathscr{M}_{s^{\prime} s^{\prime}}^{z z}} p_{z}\right) \chi_{s^{\prime}} \\
+p_{s^{\prime} c}^{z} p_{z} \chi_{c}+\frac{1}{2} p_{z} \frac{1}{\mathscr{M}_{s^{\prime} l^{\prime}}^{z z}} p_{z} \chi_{l^{\prime}}=E \chi_{s^{\prime}} .
\end{gathered}
$$

Since we are interested in 2PA well below the energy of the higher conduction bands, we do not actually need effectivemass relations for them. We just need to know their effect on the conduction-band effective mass. To that end, we rearrange as before to find

$$
\begin{aligned}
& \chi_{l^{\prime}}=\sqrt{\frac{2}{3}} P \frac{1}{E-E_{l^{\prime}}-V_{l^{\prime}}(z)} p_{z} \chi_{c}, \\
& \chi_{s^{\prime}}=-\sqrt{\frac{1}{3}} P \frac{1}{E-E_{s^{\prime}}-V_{s^{\prime}}(z)} p_{z} \chi_{c} .
\end{aligned}
$$

Finally, we look at the $\mathbf{k} \cdot \mathbf{p}$ expansion for the conduction band:

$$
\begin{aligned}
& \left(E_{c}+\frac{p_{z}^{2}}{2}+V_{c}(z)+\frac{1}{2} p_{z} \frac{1}{\mathscr{M}_{c c}^{z z}} p_{z}\right) \chi_{c}+p_{c l}^{z} p_{z} \chi_{l}+p_{c s}^{z} p_{z} \chi_{s} \\
& +p_{c s^{\prime}}^{z} p_{z} \chi_{s^{\prime}}+p_{c l^{\prime}}^{z} p_{z} \chi_{l^{\prime}}=E \chi_{c} .
\end{aligned}
$$

Plugging in the expansions in Eq. (E14) yields

$$
\frac{1}{2} p_{z} \frac{1}{m_{c}^{z}\left(E_{c}, z\right)} p_{z} \chi_{c}(z)+V_{c}(z) \chi_{c}(z)=E_{c} \chi_{c}(z),
$$

with the effective mass altered to

$$
\begin{aligned}
\frac{m_{0}}{m_{c}^{z}}= & 1+\frac{m_{0}}{\mathscr{M}_{c c}^{z z}}+\frac{2}{3} \frac{E_{p}}{E-V_{l}(z)}+\frac{1}{3} \frac{E_{p}}{E+\Delta-V_{s}(z)} \\
& +\frac{2}{3} \frac{E_{p}^{\prime}}{E-E_{g}^{\prime}-V_{l^{\prime}}(z)}+\frac{1}{3} \frac{E_{p}^{\prime}}{E-E_{s^{\prime}}-V_{s^{\prime}}(z)} .
\end{aligned}
$$

\section{APPENDIX F: INTERSUBBAND MATRIX ELEMENT}

This Appendix derives the intersubband matrix element in Eq. (17). The procedure shown is for conduction bands in the eight-band basis, but it is easily generalized to other bands and different basis sets.

The momentum matrix element between states given in the envelope expansion [Eq. (12)] is

$$
P_{j n, i m}^{z}=\left\langle\psi_{j n}, p_{z} \psi_{i m}\right\rangle=\sum_{\mu \nu}\left\langle u_{\mu} \chi_{j n}^{\mu}, p_{z} u_{\nu} \chi_{i m}^{v}\right\rangle,
$$

where we have chosen to represent inner products with operator $\hat{T}$ as $\langle f, \hat{T} g\rangle$ for clarity. As usual, $\langle\lambda f, g\rangle=\lambda^{*}\langle f, g\rangle$. We apply the chain rule for the differential operator $p_{z}$, then assume $\chi$ and $u$ change on different enough scales so that we can integrate their expressions separately. In atomic units we find

$$
\begin{aligned}
P_{j n, i m}^{z}= & \sum_{\mu \nu}\left\langle\chi_{j n}^{\mu}, p_{z} \chi_{i m}^{v}\right\rangle \delta_{\mu \nu}+p_{\mu \nu}^{z}\left\langle\chi_{j n}^{\mu}, \chi_{i m}^{v}\right\rangle \\
= & \left\langle\chi_{j n}^{c}, p_{z} \chi_{i m}^{c}\right\rangle+\left\langle\chi_{j n}^{l}, p_{z} \chi_{i m}^{l}\right\rangle+\left\langle\chi_{j n}^{s}, p_{z} \chi_{i m}^{s}\right\rangle \\
& +\sqrt{\frac{2}{3}} P\left\langle\chi_{j n}^{l}, \chi_{i m}^{c}\right\rangle-\sqrt{\frac{1}{3}} P\left\langle\chi_{j n}^{s}, \chi_{i m}^{c}\right\rangle \\
& +\sqrt{\frac{2}{3}} P\left\langle\chi_{j n}^{c}, \chi_{i m}^{l}\right\rangle-\sqrt{\frac{1}{3}} P\left\langle\chi_{j n}^{c}, \chi_{i m}^{s}\right\rangle
\end{aligned}
$$

with $p_{\mu \nu}^{z}$ obtained once again from Eq. (D1) and Table IV. Suppose now that the initial and final envelopes are conduction states. By substituting for $\chi^{l}$ and $\chi^{s}$ using Eq. (E8) we immediately find

$$
\begin{aligned}
P_{c n, c m}^{z}= & \frac{1}{2}\left[\left\langle p_{z} \chi_{n}^{c}, \chi_{m}^{c}\right\rangle+\frac{4 P^{2}}{3}\left\langle\frac{1}{E_{n}-V_{v}(z)} p_{z} \chi_{n}^{c}, \chi_{m}^{c}\right\rangle\right. \\
& \left.+\frac{2 P^{2}}{3}\left\langle\frac{1}{E_{n}+\Delta-V_{v}(z)} p_{z} \chi_{n}^{c}, \chi_{m}^{c}\right\rangle\right] \\
& +\frac{1}{2}\left[\left\langle\chi_{n}^{c}, p_{z} \chi_{m}^{c}\right\rangle+\frac{4 P^{2}}{3}\left\langle\chi_{n}^{c}, \frac{1}{E_{m}-V_{v}(z)} p_{z} \chi_{m}^{c}\right\rangle\right. \\
& \left.+\frac{2 P^{2}}{3}\left\langle\chi_{n}^{c}, \frac{1}{E_{m}+\Delta-V_{v}(z)} p_{z} \chi_{m}^{c}\right\rangle\right]
\end{aligned}
$$

where we dropped the subscript $c$ on the right-hand side. We also used the fact that $\left\langle\chi_{n}^{c}, p_{z} \chi_{m}^{c}\right\rangle=\left[\left\langle p_{z} \chi_{n}^{c}, \chi_{m}^{c}\right\rangle+\right.$ $\left.\left\langle\chi_{n}^{c}, p_{z} \chi_{m}^{c}\right\rangle\right] / 2$. Noting that $E_{p}=2 P^{2}$ in atomic units, comparison with Eq. (14) immediately leads to

$$
\begin{aligned}
\left\langle\psi_{c n}, p_{z} \psi_{c m}\right\rangle= & \frac{1}{2}\left\langle\left[\frac{1}{m\left(E_{n}, z\right)}-\frac{1}{\mathscr{M}_{c c}^{z z}}\right] p_{z} \chi_{n}^{c}, \chi_{m}^{c}\right\rangle \\
& +\frac{1}{2}\left\langle\chi_{n}^{c},\left[\frac{1}{m\left(E_{m}, z\right)}-\frac{1}{\mathscr{M}_{c c}^{z z}}\right] p_{z} \chi_{m}^{c}\right\rangle .
\end{aligned}
$$


Normalization and conversion back to bra-ket notation gives Eq. (17). This process can be repeated for hole transitions and states written in the 14-band basis.

\section{APPENDIX G: ANALYTICAL EXPRESSION FOR NORMALIZED TRANSMISSION}

The goal of this Appendix is to derive the simplest equation that can accurately describe nonlinear propagation in our system. We find that we can compute normalized transmission without accounting for linear and nonlinear pulse spreading effects. Since the pump photon energy is too low to experience nonlinear loss through $2 \mathrm{PA}$, we take the pump propagation to be linear with absorption coefficient $\sigma_{2}$,

$$
\left(\frac{\partial}{\partial x}+\beta_{1}^{(2)} \frac{\partial}{\partial t}\right) A_{2}(x, t)=-\frac{\sigma_{2}}{2} A_{2}(x, t) .
$$

The propagation direction is fixed along the $x$ direction to be consistent with the axes chosen in the paper. We can solve Eq. (G1) by transforming into the moving frame with $T=$ $t-\beta_{1}^{(2)} x$ and $X=x$. After converting back to the original coordinates, we see that the solutions must be of the form

$$
A_{2}(x, t)=F\left(0, t-\beta_{1}^{(2)} x\right) \exp \left(-\frac{\sigma_{2}}{2} x\right) .
$$

Letting $A_{2}$ be Gaussian and remembering that $\left|A_{l}\right|^{2}$ are normalized to represent instantaneous power,

$$
P_{2}=\left|A_{2}\right|^{2}=P_{02} \exp \left[-\frac{\left(t-\beta_{1}^{(2)} x\right)^{2}}{\tau_{2}^{2}}\right] \exp \left(-\sigma_{2} x\right) .
$$

Now we can plug into the equation for the probe traveling in the presence of the pump:

$$
\left(\frac{\partial}{\partial x}+\beta_{1}^{(1)} \frac{\partial}{\partial t}\right) A_{1}=2 i \gamma_{12}\left|A_{2}\right|^{2} A_{1}-\frac{\sigma_{1}}{2} A_{1} .
$$

We apply an integrating factor to remove the linear loss term by letting $A_{1}=A_{1}^{\prime} \exp \left(-\sigma_{1} x / 2\right)$. Transforming to coordinates moving with the probe group velocity $T=t-\beta_{1}^{(1)} x$ and $X=$ $x$ gives

$$
\frac{\partial A_{1}^{\prime}(X, T)}{\partial X}=2 i \gamma_{12}\left|A_{2}(X, T)\right|^{2} A_{1}^{\prime}(X, T) .
$$

We can calculate the power evolution of wave 1 by

$$
\frac{\partial}{\partial X}\left|A_{1}^{\prime}\right|^{2}=A_{1}^{\prime *} \frac{\partial A_{1}^{\prime}}{\partial X}+A_{1}^{\prime} \frac{\partial A_{1}^{* *}}{\partial X} .
$$

Substituting Eq. (G5) for the derivatives and integrating both sides over the time parameter $T$,

$$
\int_{-\infty}^{\infty} \frac{\partial P_{1}}{\partial X} d T=-4 \operatorname{Im}\left\{\gamma_{12}\right\} \int_{-\infty}^{\infty} P_{2} P_{1} d T .
$$

We have dropped the prime in $P_{1}$ terms representing the decay being factored out, but in the end the probe decay will have no effect on normalized transmission. We assume a Gaussian $P_{1}$ (probe) profile with a time delay of $\tau$ relative to the pump:

$$
P_{1}(X, T)=P_{01}(X) \exp \left[-\frac{(T-\tau)^{2}}{\tau_{1}^{2}}\right] .
$$

Substituting $t=T+\beta_{1}^{(1)}$ into the pump distribution from Eq. (G3) and plugging into Eq. (G7) along with the probe profile, we find

$$
\begin{aligned}
\frac{\partial E_{1}}{\partial X}= & -4 \operatorname{Im}\left\{\gamma_{12}\right\} P_{01}(X) P_{02} \exp \left(-\sigma_{2} X\right) \\
& \times \int_{-\infty}^{\infty} \exp \left[-\frac{(T+\rho X)^{2}}{\tau_{2}^{2}}\right] \exp \left[-\frac{(T-\tau)^{2}}{\tau_{1}^{2}}\right] d T .
\end{aligned}
$$

The temporal integral evaluates to

$$
\begin{aligned}
\frac{\partial E_{1}}{\partial X}= & -4 \operatorname{Im}\left\{\gamma_{12}\right\} P_{01}(X) P_{02} \sqrt{\pi} \frac{\tau_{1} \tau_{2}}{\tau_{x}} \\
& \times \exp \left[-\frac{(\tau+\rho X)^{2}}{\tau_{x}^{2}}-\sigma_{2} X\right],
\end{aligned}
$$

using again the GVM parameter $\rho=\beta_{1}^{(1)}-\beta_{1}^{(2)}$. We replace the probe power with its energy by applying the relation $E_{j}=$ $\sqrt{\pi} \tau_{j} P_{0 j}$, then integrate over the length of the waveguide to find the output energy:

$$
\begin{aligned}
\int_{E(0)}^{E(L)} \frac{d E_{1}}{E_{1}}= & -\frac{4}{\sqrt{\pi}} \operatorname{Im}\left\{\gamma_{12}\right\} E_{2} \frac{1}{\tau_{x}} \\
& \times \int_{0}^{L} \exp \left[-\frac{(\tau+\rho X)^{2}}{\tau_{x}^{2}}-\sigma_{2} X\right] d X .
\end{aligned}
$$

Solving the integral finally gives the result

$$
\begin{aligned}
\ln \left(\frac{E_{1}(L)}{E_{1}(0)}\right) \\
=2 \frac{E_{2} \operatorname{Im}\{\gamma\}}{\rho} \exp \left[\frac{\sigma_{2} \tau}{\rho}+\left(\frac{\sigma_{2} \tau_{x}}{2 \rho}\right)^{2}\right] \\
\quad \times\left[\operatorname{erf}\left(\frac{\tau}{\tau_{x}}+\frac{\sigma_{2} \tau_{x}}{2 \rho}\right)-\operatorname{erf}\left(\frac{\tau+\rho L}{\tau_{x}}+\frac{\sigma_{2} \tau_{x}}{2 \rho}\right)\right] .
\end{aligned}
$$

Recall that we still have an exponential decay that has been factored out of the profile $A_{1}$. We see that replacing the integrating factor gives $E_{1}\left(L, E_{2}\right)=E_{1}(0) \exp \left(-\sigma_{1} L\right) \Gamma\left(E_{2}\right)$, where $\Gamma\left(E_{2}\right)$ is the exponential of the right-hand side of Eq. (G12). Since normalized transmission is defined as $\Delta T\left(E_{2}\right)=E\left(L, E_{2}\right) / E(L, 0)$, we immediately see that the decays cancel to give $\Delta T=\Gamma\left(E_{2}\right)$.
[1] D. A. Fishman, C. M. Cirloganu, S. Webster, L. A. Padilha, M. Monroe, D. J. Hagan, and E. W. Van Stryland, Nat. Photonics 5, 561 (2011).

[2] H. S. Pattanaik, M. Reichert, D. J. Hagan, and E. W. Van Stryland, Opt. Express 24, 1196 (2016).

[3] T. K. Liang, L. R. Nunes, T. Sakamoto, K. Sasagawa, T. Kawanishi, M. Tsuchiya, G. R. A. Priem, D. Van Thourhout,
P. Dumon, R. Baets, and H. K. Tsang, Opt. Express 13, 7298 (2005).

[4] M. Reichert, A. L. Smirl, G. Salamo, D. J. Hagan, and E. W. Van Stryland, Phys. Rev. Lett. 117, 073602 (2016).

[5] S. Melzer, C. Ruppert, A. D. Bristow, and M. Betz, Opt. Lett. 43, 5066 (2018). 
[6] A. Hayat, P. Ginzburg, and M. Orenstein, Nat. Photonics 2, 238 (2008).

[7] C. N. Ironside, IEEE J. Quantum Electron. 28, 842 (1992).

[8] D. J. Gauthier, Q. Wu, S. E. Morin, and T. W. Mossberg, Phys. Rev. Lett. 68, 464 (1992).

[9] A. Hayat, A. Nevet, P. Ginzburg, and M. Orenstein, Semicond. Sci. Technol. 26, 083001 (2011).

[10] N. Poulvellarie, C. Ciret, B. Kuyken, F. Leo, and S. P. Gorza, Phys. Rev. Appl. 10, 24033 (2018).

[11] H. N. Spector, Phys. Rev. B 35, 5876 (1987).

[12] A. Pasquarello and A. Quattropani, Phys. Rev. B 38, 6206 (1988).

[13] M. Nithisoontorn, K. Unterrainer, S. Michaelis, N. Sawaki, E. Gornik, and H. Kano, Phys. Rev. Lett. 62, 3078 (1989).

[14] A. Shimizu, Phys. Rev. B 40, 1403 (1989).

[15] K. Tai, A. Mysyrowicz, R. J. Fischer, R. E. Slusher, and A. Y. Cho, Phys. Rev. Lett. 62, 1784 (1989).

[16] C. C. Yang, A. Villeneuve, G. I. Stegeman, C. H. Lin, and H. H. Lin, IEEE J. Quantum Electron. 29, 2934 (1993).

[17] A. Pasquarello and A. Quattropani, Phys. Rev. B 42, 9073 (1990).

[18] H. S. Pattanaik, M. Reichert, J. B. Khurgin, D. J. Hagan, and E. W. Van Stryland, IEEE J. Quantum Electron. 52, 1 (2016).

[19] J. B. Khurgin, J. Opt. Soc. Am. B 11, 624 (1994).

[20] S. L. Chuang, Physics of Optoelectronic Devices (Wiley, New York, 1995).

[21] C. C. Lee and H. Y. Fan, Phys. Rev. B 9, 3502 (1974).

[22] N. G. Basov, A. Z. Grasyuk, I. G. Zubarev, V. A. Katulin, and O. N. Krokhin, Zh. Eksp. Teor. Fiz. 50, 551 (1966) [JETP 23, 366 (1966)].

[23] M. Sheik-Bahae and E. W. Van Stryland, Nonlinear Optics in Semiconductors I (Elsevier Masson SAS, New York, 1999), Chap. 4, pp. 257-318.

[24] D. C. Hutchings and E. W. Van Stryland, J. Opt. Soc. Am. B 9 , 2065 (1992).

[25] J. M. Luttinger and W. Kohn, Phys. Rev. 97, 869 (1955).

[26] G. Bastard, Phys. Rev. B 24, 5693 (1981).

[27] G. Bastard, Wave Mechanics Applied to Semiconductor Heterostructures (Les Éditions de Physique, Les Ulis, 1976).

[28] P. O. Löwdin, J. Chem. Phys. 19, 1396 (1951).
[29] A. T. Meney, B. Gonul, and E. P. O'Reilly, Phys. Rev. B 50, 10893 (1994).

[30] B. S. Wherrett, J. Opt. Soc. Am. B 1, 67 (1984).

[31] J. Faist, Quantum Cascade Lasers (Oxford University Press, New York, 2013), chap. 4.

[32] N. W. Ashcroft and N. D. Mermin, Solid State Physics (Holt, Rinehart and Winston, New York, 1976).

[33] M. Yamanishi and I. Suemune, Jpn. J. Appl. Phys. 23, L35 (1984).

[34] C. Hermann and C. Weisbuch, Phys. Rev. B 15, 823 (1977).

[35] S. L. Chuang, Phys. Rev. B 43, 9649 (1991).

[36] J. Hübner, S. Döhrmann, D. Hägele, and M. Oestreich, Phys. Rev. B 79, 193307 (2009).

[37] C. M. Cirloganu, L. A. Padilha, D. A. Fishman, S. Webster, D. J. Hagan, and E. W. Van Stryland, Opt. Express 19, 22951 (2011).

[38] Y. Varshni, Physica 34, 149 (1967).

[39] M. El Allali, C. B. Sorensen, E. Veje, and P. TidemandPetersson, Phys. Rev. B 48, 4398 (1993).

[40] I. Vurgaftman, J. R. Meyer, and L. R. Ram-Mohan, J. Appl. Phys. 89, 5815 (2001).

[41] M. Cardona, K. L. Shaklee, and F. H. Pollak, Phys. Rev. 154, 696 (1967).

[42] I. Balslev, Phys. Rev. 177, 1173 (1969).

[43] P. Lawaetz, Phys. Rev. B 4, 3460 (1971).

[44] P. Pfeffer and W. Zawadzki, Phys. Rev. B 53, 12813 (1996).

[45] R. Eppenga, M. F. H. Schuurmans, and S. Colak, Phys. Rev. B 36, 1554 (1987).

[46] Q. H. F. Vrehen, J. Phys. Chem. Solids 29, 129 (1968).

[47] D. E. Aspnes and A. A. Studna, Phys. Rev. B 7, 4605 (1973).

[48] P. P. Harrison and A. Valavanis, Quantum Wells, Wires and Dots, 2nd ed. (Wiley, Sussex, England, 2005).

[49] S. Adachi, J. Appl. Phys. 66, 6030 (1989).

[50] Lumerical Inc., https://www.lumerical.com/products/.

[51] Q. Lin, O. J. Painter, and G. P. Agrawal, Opt. Express 15, 16604 (2007).

[52] G. P. Agrawal, Nonlinear Fiber Optics (Academic, New York, 2012), p. 631

[53] E. O. Kane, J. Phys. Chem. Solids 1, 249 (1957).

[54] P. Y. Yu and M. Cardona, Fundamentals of Semiconductors (Springer, New York, 2010). 